\title{
UAV based distributed automatic target detection algorithm under realistic simulated environmental effects
}

\author{
Shanshan Gong \\ West Virginia University
}

Follow this and additional works at: https://researchrepository.wvu.edu/etd

\section{Recommended Citation}

Gong, Shanshan, "UAV based distributed automatic target detection algorithm under realistic simulated environmental effects" (2007). Graduate Theses, Dissertations, and Problem Reports. 1824.

https://researchrepository.wvu.edu/etd/1824

This Thesis is protected by copyright and/or related rights. It has been brought to you by the The Research Repository @ WVU with permission from the rights-holder(s). You are free to use this Thesis in any way that is permitted by the copyright and related rights legislation that applies to your use. For other uses you must obtain permission from the rights-holder(s) directly, unless additional rights are indicated by a Creative Commons license in the record and/ or on the work itself. This Thesis has been accepted for inclusion in WVU Graduate Theses, Dissertations, and Problem Reports collection by an authorized administrator of The Research Repository @ WVU. For more information, please contact researchrepository@mail.wvu.edu. 


\title{
UAV Based Distributed Automatic Target Detection Algorithm under Realistic Simulated Environmental Effects
}

\section{Shanshan Gong}

\author{
A Thesis submitted to the \\ College of Engineering and Mineral Resources \\ at West Virginia University \\ in partial fulfillment of the requirements \\ for the degree of \\ Master of Science \\ in \\ Electrical Engineering \\ Natalia A. Schmid, D.Sc., Chair \\ Matthew C. Valenti, Ph.D. \\ Xin Li, Ph.D. \\ Lane Department of Computer Science \\ and Electrical Engineering \\ Morgantown, West Virginia \\ 2007
}

Keywords: Automatic Target Detection, Data Fusion, Swarmed UAV

(c) Copyright 2007 by Shanshan Gong

All Rights Reserved 


\section{ABSTRACT \\ UAV Based Distributed Automatic Target Detection Algorithm under Realistic Simulated Environmental Effects \\ Shanshan Gong}

Over the past several years, the military has grown increasingly reliant upon the use of unattended aerial vehicles (UAVs) for surveillance missions. There is an increasing trend towards fielding swarms of UAVs operating as large-scale sensor networks in the air[1]. Such systems tend to be used primarily for the purpose of acquiring sensory data with the goal of automatic detection, identification, and tracking objects of interest. These trends have been paralleled by advances in both distributed detection [2], image/signal processing and data fusion techniques[3]. Furthermore, swarmed UAV systems must operate under severe constraints on environmental conditions and sensor limitations. In this work, we investigate the effects of environmental conditions on target detection performance in a UAV network. We assume that each UAV is equipped with an optical camera, and use a realistic computer simulation to generate synthetic images. The automatic target detector is a cascade of classifiers based on Haar-like features. The detector's performance is evaluated using simulated images that closely mimic data acquired in a UAV network under realistic camera and environmental conditions. In order to improve automatic target detection (ATD) performance in a swarmed UAV system, we propose and design several fusion techniques both at the image and score level and analyze both the case of a single observation and the case of multiple observations of the same target. 


\section{Acknowledgements}

First, I would like to thank Dr. Natalia Schmid for being such a patient and understanding thesis advisor. Her foresight, intuition, and care were instrumental in shaping this work. I have learned so much from her since I joined the Statistical Signal Processing Lab at West Virginia University. I also would like to thank my graduate committee members Dr. Xin Li and Dr. Matthew Valenti for their expert advice and support to my study and thesis.

I must thank Xiaohan for her seemingly infinite supply of ideas and support for this work. I also thank Jinyu, Nathan and Francesco for their support and discussion which helped me so much on my study and research. Lastly, I thank my parents and my boyfriend Lei for always supporting my choice.

If I may, I would also like to take this moment to thank many great teachers, mentors and friends that I have had the pleasure to interact with over the past two years. 


\section{Contents}

Acknowledgements $\quad$ iii

1 Introduction 1

1.1 Background and Motivation . . . . . . . . . . . . . 1

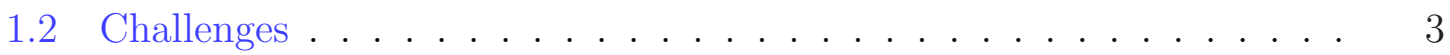

1.3 Literature Review . . . . . . . . . . . . . . . . . . . . . 4

1.3.1 Swarmed UAVs . . . . . . . . . . . . . . . . . 4

1.3.2 Automatic Target Detection . . . . . . . . . . 5

1.3.3 Data Fusion . . . . . . . . . . . . . . . 7

1.4 Organization .......................... 8

2 Single-frame Automatic Target Detection 10

2.1 Haar-like Features . . . . . . . . . . . . . . . . . . . . . . 11

2.2 AdaBoost Learning . . . . . . . . . . . . . . . . . . . . . . . 12

2.3 Classifier Cascade . . . . . . . . . . . . . . . . . . . 14

2.4 Performance Evaluation . . . . . . . . . . . . . . . . . 14

3 Multi-frame Automatic Target Detection 17

3.1 Image-level Data Fusion for Improved ATD . . . . . . . . . . . . . . . 18

3.1.1 Super-resolution for Improved ATD . . . . . . . . . . . . . 18

3.1 .2 Image Mosaicking for Improved ATD . . . . . . . . . . . . . 21

3.2 Score-level Data Fusion for Improved Detection . . . . . . . . . . . . 22 
4 Numerical Results $\quad 25$

4.1 Database Description . . . . . . . . . . . . . . 25

4.1.1 Simulated Optical Data Set . . . . . . . . . . . 25

4.1.2 Simulated Environmental and Camera Distortions . . . . . . . 27

4.1.3 Data for Testing the Effect of Occlusion . . . . . . . . . . . 29

4.2 Results: Single-frame Detector . . . . . . . . . . . . . . . 31

4.2.1 Learning Results of Single-frame Detector . . . . . . . . . . . 31

4.2.2 Influence of Environmental and Camera Effects on Detection Performance ..................... . . 31

4.2.3 Influence of Occlusion on Detection Performance . . . . . . . . 32

4.3 Results: Multiple-frame Detector . . . . . . . . . . . . . 33

4.3.1 Detection Performance: Super-Resolution for Improved ATD . 33

4.3.2 Detection Performance: Image Mosaicking for Improved ATD 35

4.3.3 Detection Performance: Score-level Data Fusion . . . . . . . . 35

5 Conclusion and Future Work 44

$\begin{array}{ll}\text { Bibliography } & 48\end{array}$ 


\section{List of Tables}

2.1 The AdaBoost Algorithm for Classifier Learning [4] . . . . . . . . . . 16

4.1 Training Parameters of Single-frame Detector . . . . . . . . . . . . 31

4.2 Summary on High Resolution and Low Resolution Detectors used in our experiments . . . . . . . . . . . . . . . . . . . 34 


\section{List of Figures}

2.1 Extended integral feature set [5]. The sum of the pixels which lie within the white rectangles are subtracted from the sum of pixels in the black rectangles. . . . . . . . . . . . . . . . . 11

2.2 Stage classifier. . . . . . . . . . . . . . . . . . 13

2.3 Cascade of classifiers. . . . . . . . . . . . . . . . . . . . 14

3.1 Basic Premise for Super Resolution. . . . . . . . . . . . . . . . . . . . 19

3.2 Super-Resolution Observation Model. . . . . . . . . . . . . . . . 20

3.3 A Block-diagram of the Interpolation-based Approach. . . . . . . . . 21

4.1 Model for Capturing Images. . . . . . . . . . . . . . . . . . . . . . . . 26

4.2 The GUI of the ATR training tool. . . . . . . . . . . . . 26

4.3 Example of Target Images Used for Training. . . . . . . . . . . . . . . 27

4.4 Example of Non-target Images Used for Training. . . . . . . . . . . . 28

4.5 Example of Testing Images. . . . . . . . . . . . . . . . . . . . 28

4.6 Examples of Distorted Testing Images. . . . . . . . . . . . . . . 36

4.7 Examples of Targets Occluded by a Single Block. . . . . . . . . . . . 37

4.8 Examples of Targets Occluded by Multiple Blocks. . . . . . . . . . . . 38

4.9 Examples of Targets Occluded by Trees. . . . . . . . . . . . . . . . 39

4.10 Single-frame Detector Features Selected by AdaBoost (First Four Stages). 39

4.11 (a)-(e) Detection performance as functions of various environmental and camera effects. . . . . . . . . . . . . . . . . . . . 40

4.12 (a)-(e) Performance of Occluded Target Detection. . . . . . . . . . 41

4.13 Example for Super-resolved Natural Image. . . . . . . . . . . . . . . 42 
4.14 Image-level Data Fusion Results. . . . . . . . . . . . . . . . . . . 43

4.15 Example for Image Mosaicking. . . . . . . . . . . . . . . . . . . . 44

4.16 Example Frames for Score-level Data Fusion. . . . . . . . . . . . . . . 45

4.17 Score-level Data Fusion Results. . . . . . . . . . . . . . . . . . 45 


\section{Chapter 1}

\section{Introduction}

\subsection{Background and Motivation}

Automatic target recognition (ATR) involves two main tasks: target detection and target recognition [6]. The purpose of automatic target detection (ATD) is to find regions of interest (ROI) where a target may be located. By locating ROIs, we can filter out a large amount of background clutter from the terrain scene, making object recognition feasible for large data sets. The ROIs are then passed to a recognition algorithm that identifies targets [6]. Automatic target detection is one of the most critical steps in the ATR problem, since the results of postprocessing depend critically on this step.

$\mathrm{ATD} / \mathrm{R}$ is performed for the purpose of surveillance, during rescue missions, or during military missions. Sensors positioned on the ground, installed on airplanes, helicopters, ground vehicles, etc acquire sensory data. Then the data then have to be processed using automatic detection and recognition algorithms. One of the most secure (especially during military mission) means of acquiring sensory data involves remotely operated vehicles. Remotely operated vehicles can be broadly divided into two categories: unmanned aerial vehicles (UAVs) and unmanned ground vehicles (UGVs). In this thesis, we focus on a distributed network of air-borne UAVs used to detect and recognize ground targets.

UAVs traditionally acquire sensory data and send the data to a central location 
such as a base station, where potential targets are identified using image analysis algorithms [7][8][9]. However, this centralized model for ATD/R possesses a number of drawbacks such as scalability and network delays in communication with the central location [10].

In developing the next generation of UAVs, one of the ideas is to utilize reactive agents and the associated swarming behavior as part of the command and control system for a group of UAVs functioning cooperatively and independently from ground control. Previous works [10][11][12] demonstrate that this technique provides a suitable mechanism to assimilate the capabilities of individual UAVs into a group of coordinating UAVs that perform $\mathrm{ATD} / \mathrm{R}$ in a distributed manner. In a swarmed system, multiple mobile entities are directed to converge on a single point of interest, disperse and regroup again. To achieve distributed ATD/R using swarming, each UAV individually searches for potential targets within an area of interest using its image sensor. As soon as the image of an object is sensed to be a possible target by a UAV, other UAVs cooperate with it by swarming towards the potential target to collectively perform $\mathrm{ATD} / \mathrm{R}$ in a data fusion manner and confirm the object as a target.

In contrast to a centralized model for ATD/R, a distributed ATD/R model possesses the potential to provide minimal user intervention, a high level of robustness, and largely autonomous operation. The distributed ATD/R model can: (1) scale up efficiently in the number of UAVs deployed and in the amount of data collected and processed for ATD/R, (2) improve the efficiency of the system because most computation is performed locally within each UAV, which in turn reduces the time required to upload every image data from different UAVs onto the central location, and, (3) reduce the communication between the central location and individual UAVs so that the system is less susceptible to the loss of data and failures due to wireless communication problems between an individual UAV and the central location [10].

In this thesis, we explore the possibility of distributed optical camera-based ATD in a swarmed UAVs system. 


\subsection{Challenges}

Automatic detection of real-world targets poses many challenging problems. An ideal detector must be able to differentiate any instantiation of the target class from everything else in the world. More specifically, it has to accommodate all possible variations of the target's appearance, e.g. with respect to color, texture, pose, scale, and illumination and at the same time be highly specific to avoid confusion with complex background clutter. A more general difficulty is the geometric ambiguity which arises from projecting three dimensions of the world onto two dimensions in the image.

Designers of UAV-based automatic target detection (ATD) systems face numerous challenges. UAVs must operate under severe communication constraints, varying environmental conditions and sensor limitations. Targets can present an infinite variety of appearances due to changes in pose and differences in illumination (in visual systems) and thermodynamic state (in infrared systems). Non-ideal sensor effects further complicate matters, such as the noise and blurring present in optical systems. In this thesis, we focus on the case where the acquired sensory data are in the form of optical images. Traditionally, optical cameras are low in cost and small in size, which makes them a high preference imagery sensors for a variety of military and civilian applications. The major limitation of optical cameras is their inability to deal with environmental conditions and imperfect camera setup which lowers fidelity of the results in the ATD task.

In a distributed UAV system, ATD/R techniques used to process images are more complex than in a centralized UAV system. In a distributed ATD system, images are first processed locally on board each UAV. Except when the target's location is obvious, decisions are not made on the basis of a single image. Rather, images are fused across time and space by using not only multiple images from a single UAV, but the images from multiple UAVs across a portion or sometimes the entire network. The UAVs share measurements of mutual information which requires a minimal amount of data to be transferred among the UAVs. Rather than sending actual images, which can be a very expensive activity in terms of usage of communication channels, the 
UAVs exchange maximally processed and compressed information required to detect and recognize targets. The information that each UAV possesses about a potential target is further fused with information received from other UAVs. Thus, besides observation noise, environmental effects, and background clutter which prevent the system from being entirely reliable, the biggest factor in determining reliability is the data fusion strategy which eventually affects the detection performance of the whole system.

\subsection{Literature Review}

This section briefly summarizes past and ongoing research in the field of optical ATD and fusion methods for improved ATD. First, we will give a general overview of swarmed UAV networks. We will further present a summary of automatic target detection and data fusion algorithms which are applied to optical data.

\subsubsection{Swarmed UAVs}

The swarming mechanism for ATD/R is based on the stigmergetic activity of social insects such as ants [11] to locate food. Stigmergy is a reinforcement learning mechanism that enables ants to indirectly communicate with each other about their environment using a chemical substance called pheromone. For example, while searching for food ants start from their nest and leave behind a pheromone trail along the path they traverse. The path leading from the nest to the food receives the highest amount of pheromone. Pheromone provides positive reinforcement to future ants, and, ants searching for the food later on use the trail as a positive reinforcement to reach the location of the food.

This mechanism can be applied to artificial systems as well. In a swarmed UAVs system, a swarm of UAVs controlled by reactive agents is employed to achieve automatic target recognition. The ideology behind the swarm concept is that a system of many simple, expendable units can attain the performance level of a small number of complex aircrafts at a lower cost. To accomplish this, the UAVs must be autonomous 
and cooperate without constant communication or direction from a ground controller.

Following the pheromone concept, each agent carries in its memory a map that stores levels of digital pheromone in the environment, and the agents can exchange map information when they pass close to each other [10]. Maintaining the digital pheromone map involves increasing the numeric value at the units current location and slowly decreasing levels across the entire map. Pheromone decay ensures that areas are revisited over time so that any changes can be detected.

Besides mapping visited areas, reactive agents may also be given a scenario map that outlines specific areas that are of higher interest than others. Similar to a digital pheromone map, the area of interest map contains constant numeric values based on what type of region is represented [10]. Priority search areas contain values that tend to attract units to those locations, while no-fly zones or known threats will cause a repellant force on nearby UAVs.

\subsubsection{Automatic Target Detection}

It is important to contrast detection with the problem of recognition where the goal is to identify specific instances of a class. A target detection system knows how to differentiate targets from everything else, while a target recognition system knows the difference between target $\mathrm{A}$ and target B. A typical detection-style algorithm scans the input image using a subwindow at all positions and scales by classifying each possible subwindow independently. It then reports the number, positions and sizes of found targets.

Automatic target detection approaches can be classified into three major categories: feature invariant approach, template matching approach and learning-based approach. In feature invariant approach, the algorithms aim to find structural features such as edges [13], textures [14], etc. that exist even when the pose, viewpoint, or lighting conditions vary, and then use the features to locate targets.

The second category consists of algorithms that attempt to match pre-defined template to different parts of the image in order to find a fit. Initial work on the detection of rigid objects in static images, such as street signs or faces, Betke et 
al. [15] and Yuille et al. [16] used this approach with a set of rigid templates or hand-crafted parameterized curves. These kinds of methods are difficult to extend to more complex objects such as people, since they involve a significant amount of prior information and domain knowledge.

The final object detection approach is characterized by its learning-based algorithms. These algorithms learn the salient features of a class from sets of labeled positive and negative examples. There are two essential issues to build such detection algorithms. First, features are extracted from the image and the object of interest is encoded using those features. Such feature selection techniques include wavelets [17], Principal Component Analysis (PCA) [18], etc. Second, a classifier is learned using these features. Popular techniques for building classifiers include Support Vector Machines (SVM) [19], neural networks [20] and boosting [21]. One of the successful systems in the area is the pedestrian detection system of Papageorigious et al. [22]. Their system detects the full body of a person. Haar wavelets are used to represent the images and Support Vector Machines (SVM) classifiers are used to classify the patterns. The system has been improved in [23], detecting pedestrians through the detection of four components of the human body. Another successful example is the face detection system from Rowley et al. [20], which consists of an ensemble of neural networks and a module to reduce false detection. Similar object detection system have been developed by others (Vaillan et al. [24], Moghaddam et al. [25] and Viola et al. [4]).

In this work, the basic target detector is the modified version of Viola-Jones detector which was originally developed for face detection [4]. The detector is a cascade of classifiers based on Haar-like features. The approach is well-known for its high accuracy and speed. The Viola-Jones classifier combines the following three strategies for speed: (1) fast to compute features; (2) classification based on simple linear thresholds, reminiscent of detection stumps in decision trees; (3) a cascaded detector whose cascade structure is learned during training.

In Viola-Jones' algorithm, all features are computed as differences of pixel sums (i.e. integrals) over rectangular regions. These features are simple in the sense that they only capture horizontal and vertical bars and edges. On the other hand, they 
can be computed in constant time independent of size and position with the help of summed area tables ("integral images"). As a result, computing these features at all scales and positions is faster than computing an image pyramid in some traditional methods [26][20].

AdaBoost [27] is used both to select features and to train the actual classifier. AdaBoost is a greedy iterative fitting procedure that in each round selects the feature which best classifies the training data. It then reweighs the training set assigning high weights to misclassified instances. AdaBoost provably drives the training error to zero exponentially in the number of rounds and at the same time achieves large margins rapidly [28]. However, with each round it also slows down the classifier by increasing its complexity.

Hence, instead of training one monolithic slow-to-evaluate classifier, Viola et al. propose an algorithm for learning a classifier cascade [29] where each cascade stage is trained using AdaBoost. A cascade allows to shortcut the computation for almost all negative test instances and only compute all features for the most promising test candidates. An exhaustive search over a single image typically means examining several tens of thousands of candidate window where a few correspond to targets.

The Viola-Jones detector inspires a large number of follow-up papers from other authors many of which use other feature types [30] or extend the original feature set [5]. Others employ variants of the boosting procedure, like FloastBoost [31], LogitBoost or Gentle AdaBoost to mitigate its greediness. Applications include profile detection of faces, lip tracking, banner (commercials) detection [5] and multi-view face detection [32]. However, the approach has not been reported to work for target (car, tank, etc.) detection in the ATR community.

\subsubsection{Data Fusion}

Combining the results of multiple sensors can provide more accurate information than using a single sensor [33][34]; this allows either improved accuracy from existing sensors or the same performance from smaller or cheaper sensors. Swarms of sensors can facilitate detecting low signal-to-clutter targets by allowing correlation between 
different aspect angles and time instances. Multi-Sensor Data Fusion (MSDF) is used in many diverse fields, such as military target detection, recognition and tracking.

MSDF system can be characterized by levels [35]: signal, pixel, feature and decision-level. The first level (called signal level) concerns with the aggregation of raw data provided directly from sensors, without any transformation. Pixel or image level fusion [36] creates new images that are more suitable for the purposes of object detection and recognition. The next fusion method is feature level fusion [37]. The raw data are first encoded (features are extracted) before being aggregated. Finally, the highest abstraction level corresponds to the decision fusion [38]. It combines decisions proposed by classifiers/detectors.

\subsection{Organization}

In this work, we explore the feasibility of distributed automatic target detection in a swarmed UAVs system. We develop a target detector for UAV-based target detection using optical images. The detector is a cascade of classifiers based on Haarlike features. As swarmed UAV systems must operate under severe constraints on environmental conditions and sensor limitations, we focus on exploring limitations and limits of our detector. We evaluate our detector performance under different environmental conditions and camera effects. A few scenarios including degraded data (lighting, contrast, Gaussian noise, motion blur, off-focus blur), occlusions and low resolution data are considered.

In order to improve the state of the art in automatic target detection, we explore the possibility of using data fusion techniques for improved ATD. We propose and design several data fusion techniques for different scenarios. In the first scenario, a super-resolution approach is used to fuse data at the image level in order to improve detection performance on a low-resolution database. In the second scenario, an image mosaicking method is employed to solve the difficulties in target detection with partial occlusion. And a score-level data fusion technique focuses on improved detection performance in a more general and common scenario.

The remainder of the thesis is organized as follows. 
Chapter 2 develops a target detector using single frame optical image information. The detector is a modified version of Viola-Jones face detector. This chapter describes the theory and details of the approach.

Chapter 3 proposes several data fusion approaches for improved automatic target detection from multiple frames. We investigate both image-level and score-level data fusion techniques under different scenarios. This chapter describes the theory and algorithms for those approaches.

Chapter 4 provides details on implementations and numerical results of our experiments. We first describe a database generation. After that, we evaluate our single-frame detector on different versions of database. Furthermore, we demonstrate the improvements of detection performance due to data fusion techniques.

Chapter 5 draws conclusions and presents opportunities for future research. 


\section{Chapter 2}

\section{Single-frame Automatic Target Detection}

Images contain a large amount of redundant information that can be partially recovered through image encoding. Image encoding will also allow the compact representation of useful information contained in the image. Under useful information we understand information that leads to efficient differentiations of objects.

Compact representation of information about objects present in images is critical for our application. Earlier we indicated that distributed airborne UAV networks have limited communication bandwidth. Thus, maximal processing of data has to be performed on board of individual UAV. Apart from this, the processing has to be performed in real time. This motivated us to select Viola and Jones Haar-like feature encoding method [4] as a target detection algorithm. This algorithm is simple and computationally efficient. The OpenCV [39] version of the algorithm is able to process 30 - 50 frames per second.

In this chapter, we explain the theory behind the algorithm and summarize the most important facts about the integral image features, AdaBoost and cascade learning. The details of implementation will be further discussed in Section 4.2.1. Then, Section 4.2.2 will present the results of detector evaluation on synthesized data.

The rapid target detection scheme is based on the idea of a boosted classifier cascade [4] but extends the original feature set and offers different boosting variants 
for learning [5]. The classifier cascade is trained on a set of positive images (targets) and a set of negative images (non-targets). For each training image, an over-complete set of Haar-like feature pool is calculated and AdaBoost algorithm of Schapire and Singer [27] is used to build a stage classifier. After the classifier cascade is trained, the detection algorithm is applied to a query image. A search window is slid over the query image. At each window location and scale, the content of the window is classified as target or non-target.

\subsection{Haar-like Features}

Haar-like features of the detector are weighted differences of integrals over rectangular subregions. Fig. 2.1 presents Lienhart's extended set of available Haar-like feature types where black and white rectangles correspond to positive and negative weights, respectively. The feature types consist of four different edge features, eight line features and two center-surround features.
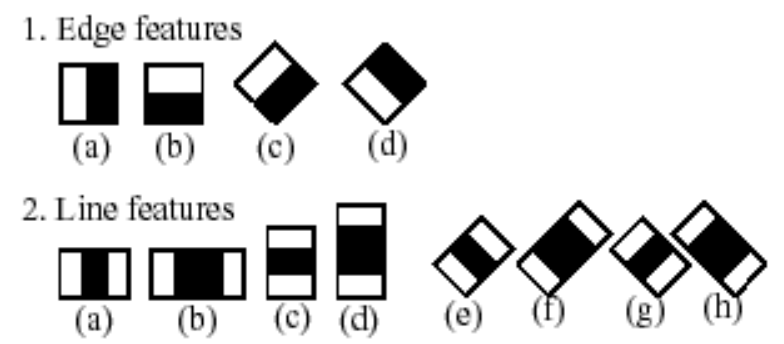

3. Center-surround features

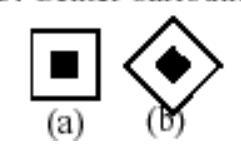

Figure 2.1: Extended integral feature set [5]. The sum of the pixels which lie within the white rectangles are subtracted from the sum of pixels in the black rectangles.

These features are reminiscent of Haar wavelets and early features of the human visual pathway such as center-surround and directional responses [40]. Their main advantage is that they can be computed in constant time at any scale. Denote the pixel sum of a rectangle $r$ as $\operatorname{RecSum}(r)$. The feature set is then the set of all possible 
features of the form

$$
\text { feature }_{I}=\sum_{i \in I=\{1, \ldots, N\}} w_{i} \cdot \operatorname{RecSum}\left(r_{i}\right)
$$

with weights $w_{i} \in \Re$, rectangles $r_{i}$ and their number $N$. Only weighted combinations of pixel sums of two rectangles are considered, that is, $N=2$. The weights have opposite signs (indicated as black and white in the figure), and are used to compensate between differences in area. Efficient computation is achieved by using summed area tables. Rotated features and center-surround features have been added to the original feature set of Viola-Jones by Lienhart et al [5] using rotated summed area tables.

\section{$2.2 \quad$ AdaBoost Learning}

Given a feature set and a training set of positive and negative images, various machine learning approaches could be used to learn a classification function. For our target detector, we use boosting as our basic classifier. Boosting is a powerful learning concept. It combines the performance of many "weak" classifiers to produce a powerful 'committee' [28]. A weak classifier is only required to be better than chance, and thus can be very simple and computationally inexpensive. Many of them efficiently combined, however, result in a strong classifier, which often outperforms most 'monolithic' strong classifiers such as Support Vector Machine (SVM) and neural networks [40].

Various boosting algorithms such as Discrete AdaBoost, Real AdaBoost and Gentle AdaBoost [21] could be used to train the classifier. All of them are identical with respect to computational complexity from a classification perspective, but differ in their learning approaches. In this work, we chose to use Gentle AdaBoost which outperforms the other two boosting algorithm from previous studies [5].

Table 2.1 illustrates the Adaboost learning algorithm. For two class problems, we are given a set of $N$ labeled training examples $\left(x_{1}, y_{1}\right), \ldots,\left(x_{N}, y_{N}\right)$, where $y_{i} \in$ $\{-1,+1\}$ is the class label associated with example $x_{i}$. For object detection, $x_{i}$ is an image sub-window of a fixed size containing an instance of the object of interest 
$\left(y_{i}=+1\right)$ or object of no interest $\left(y_{i}=-1\right)$.

In each round of boosting, a single rectangle feature which best separates the positive and negative samples is selected by the learning algorithm. For each feature, the weak learner determines the optimal threshold classification function, such that the minimum number of examples are misclassified. Thus, a weak classifier $h_{j}(\cdot)$ is a binary valued function obtained by comparing the $j$-th feature value $f_{j}(\cdot)$ with a threshold $\theta_{j}$ :

$$
h_{j}(x)=\left\{\begin{array}{cc}
\alpha_{j} & \text { if } f_{j}(x)>\theta_{j} \\
\beta_{j} & \text { otherwise, }
\end{array}\right.
$$

where $\theta_{j}$ is the optimal threshold obtained by the weak learner. Here $x$ is a subwindow of an image. The value of the feature is equal to weighted differences of integrals over rectangular subregions. $\alpha_{j}$ and $\beta_{j}$ are positive or negative votes of each feature set by AdaBoost during the learning process.

The form of the final stage classifier returned by AdaBoost is a thresholded linear combination of weak classifiers (see Fig. 2.2). The stage classifier is given by:

$$
C(x)=\left\{\begin{array}{c}
1, \quad \text { if } \sum_{j} h_{j}(x)>T, \\
0, \quad \text { otherwise }
\end{array}\right.
$$

where $T$ is the stage threshold set by AdaBoost during the learning process.

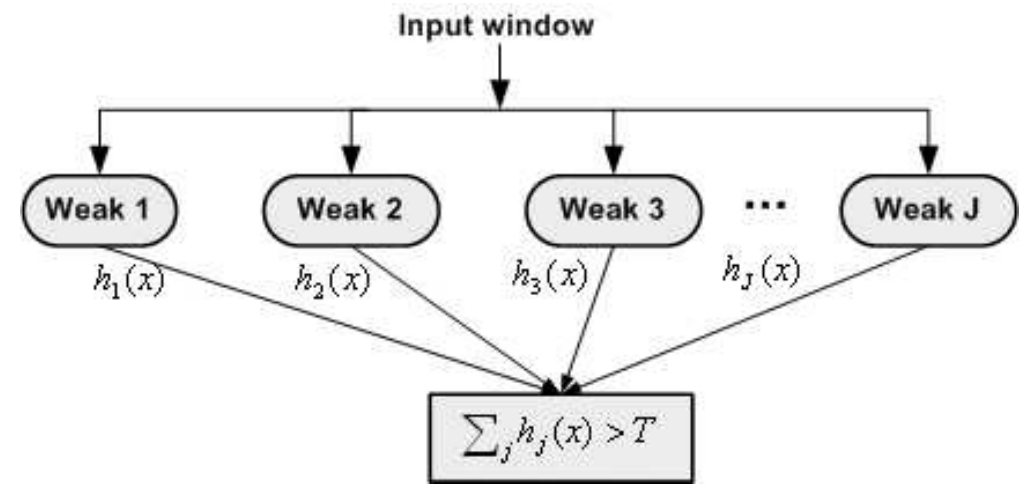

Figure 2.2: Stage classifier. 


\subsection{Classifier Cascade}

In order to improve computational efficiency and also reduce the false positive rate, a sequence of increasingly more complex classifiers called cascade is used. A cascade of classifiers is a degenerated decision tree. At each stage almost all objects of interest are detected while only a certain fraction of the non-object patterns are rejected. In our case each stage was trained to eliminate $50 \%$ of the non-target patterns while falsely eliminating only $0.1 \%$ of the target patterns; 14 stages were trained. Assuming that our test set is a representative for the learning task, we can expect a false alarm rate about $0.5^{14} \approx 6.1 E-05$ and a hit rate about $0.999^{14} \approx 0.98$.

The more an input window looks like an object, the larger the number of classifiers are evaluated on it and the longer it takes to classify the window. Since most windows of an image do not look like objects, they are quickly discarded as non-objects. Fig. 2.3 illustrates a cascade.

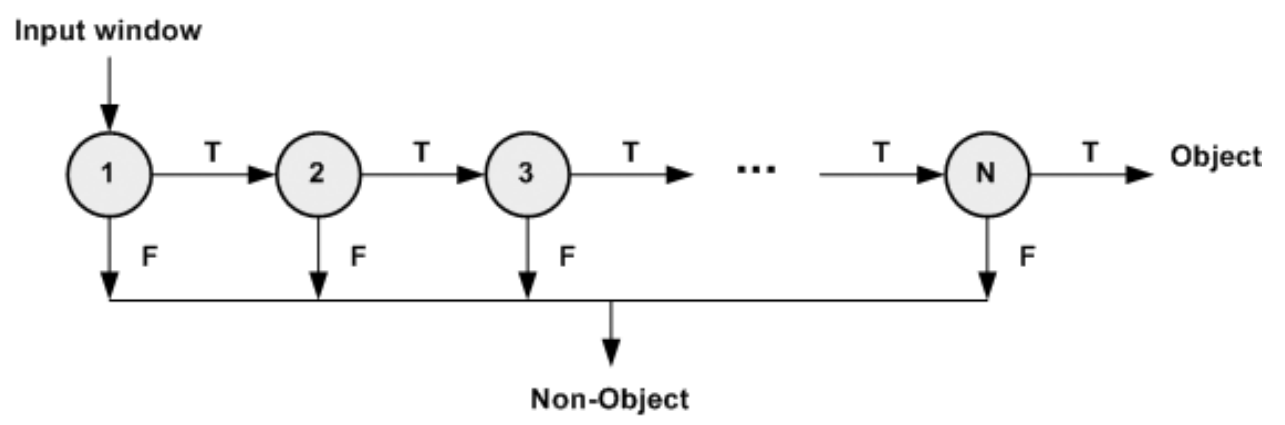

Figure 2.3: Cascade of classifiers.

\subsection{Performance Evaluation}

To evaluate detection performance, we involve Receiver Operating Characteristic (ROC) curves. There are two ways to construct ROC curves.

During detection, multiple targets are often detected near by the location and at the scale of an actual target location. Therefore, it would be more appropriate to merge multiple detection results. ROCs are constructed by varying the required 
number of detected targets per actual target before merging into a single detection result. The method is employed by the OpenCV. In our experiments, we found that the ROCs generated by this method are relatively smooth.

In order to have a more reasonable evaluation of detector's performance, we developed another way to generate the ROCs. In this approach, the detection threshold is selected as the threshold of the final classifier stage. Adjusting the threshold to $+\infty$ will yield a detection rate of 0.0 and a false positive rate of 0.0 . Adjusting the threshold to $-\infty$, however, increases both the detection rate and false positive rate, but only to a certain point. In fact, a threshold of $-\infty$ in the final stage is equivalent to removing that layer. Further increasing the detection and false positive rates requires decreasing value of the threshold of the next classifier in cascade. Thus, in order to construct a complete ROC curve, classifier layers are removed one by one. We use the number of false positives as opposed to the rate of false positive to label the $x$-axis. The false positive rate can be calculated by simply dividing the number of false positives by the total number of scanned sub-windows. 
Table 2.1: The AdaBoost Algorithm for Classifier Learning [4]

1. Given example images $\left(x_{1}, y_{1}\right), \ldots,\left(x_{n}, y_{n}\right)$ where $y_{i}=0,1$ for negative and positive examples respectively.

2. Initialize weights $w_{1, i}=\frac{1}{2 m}, \frac{1}{2 l}$ for $y_{i}=0,1$ respectively, where $m$ and $l$ are the number of negative and positive respectively.

3. For $t=1, \ldots, T$ :

(1) Normalize the weights,

$$
w_{t, i} \leftarrow \frac{w_{t, i}}{\sum_{j=1}^{n} w_{t, j}}
$$

so that $w_{t}$ is a probability distribution.

(2) For each feature, $j$, train a classifier $h_{j}$ which is restricted to using a single feature. The error is evaluated with respect to $w_{t}, \epsilon_{j}=\sum_{i} w_{i}\left|h_{j}\left(x_{i}\right)-y_{i}\right|$.

(3) Choose the classifier, $h_{t}$, with the lowest error $\epsilon_{t}$.

(4) Update the weights:

$$
w_{t+1, i}=w_{t, i} \beta_{t}^{1-e_{i}}
$$

where $e_{i}=0$ if example $x_{i}$ is classified correctly, $e_{i}=1$ otherwise, and $\beta_{t}=\frac{\epsilon_{t}}{1-\epsilon_{t}}$ 4. The final strong classifier is:

$$
h_{(x)}=\left\{\begin{array}{c}
1 \sum_{t=1}^{T} \alpha_{t} h_{t}(x) \geq \frac{1}{2} \sum_{t=1}^{T} \alpha_{t} \\
0 \quad \text { otherwise }
\end{array}\right.
$$

where $\alpha_{t}=\log \frac{1}{\beta_{t}}$ 


\section{Chapter 3}

\section{Multi-frame Automatic Target Detection}

In this chapter, we motivate and describe several data fusion methods for improved detection performance. Swarms of sensors can facilitate detecting targets by allowing correlation between different aspect angles and time instances. In a sense, a collection of sensors positioned on individual UAV is equivalent to broadening the aperture, which can be exploited to yield much better detection and discrimination performance.

Consider a scenario where a set of UAVs perform an area search. UAVs monitor the ground continuously at a slow rate (for instance, $2-5$ frames per second). We assume that a UAV while passing a target is capable of acquiring only a few $(1-4$ frames) containing this target. Now, if a UAV detects a potential target within a frame, it may appeal to its neighbors to perform additional monitoring of the area. Thus, this scenario may result in collecting a relatively large number of optical frames containing information about a target.

Here we assume three kinds of situations for which different levels of data fusion techniques are applied to achieve improved ATD. First, if a target image is acquired at a low resolution (due to high altitude flight or absence of zoom), a single frame-based detection provides poor results. However, if a set of frames containing information about the same target are available, the detection performance may be improved 
considerably due to use of a super-resolution (SR) technique (Section 3.1.1). Second, if targets are occluded by clutter, a single frame-based detection may fail. In such a case, image mosaicking techniques could be applied to assemble information contained in images at the same target but acquired at different view angles. We will further show that this improves the detection performance (Section 3.1.2). We also explore data fusion techniques for images with a sufficient resolution. In the first two situations, image-level data fusion techniques are employed, while in the last one a score-level data fusion technique (Section 3.2) is used.

\subsection{Image-level Data Fusion for Improved ATD}

\subsubsection{Super-resolution for Improved ATD}

\section{Scenario and Assumption}

In our swarmed UAV system, image data is gathered by charge coupled device (CCD) cameras which are mounted on each UAV. Such images suffer from non-ideal sensor effects such as shot noise and blurring effects present in the optical system. The image resolution is also inherently limited by the detection array used to capture the image. Moreover, in some situations, the UAVs are restricted to fly at a high altitude (1000 - 1500 feet) which would result in gathering low-resolution images. All of these low-resolution images could not provide sufficient details for advanced image processing operations such as automatic target detection and recognition.

Fortunately, if a set of frames captured by multiple neighboring UAVs containing information about the same target, image super-resolution (SR) techniques [41] can

be applied to overcome the limits of imaging system. Low-resolution images can be fused to yield an image of a higher resolution compared to any original low resolution frames. This increase in resolution can have potentially dramatic consequences for improved ATD on the resultant higher-resolution images. 


\section{Image Super-Resolution Model}

The field of image super-resolution (SR) arose from the need to overcome the inherited resolution limitation of low-resolution (LR) imaging systems to generate higherresolution images. Image super-resolution has been one of the most active research area in the field of image processing and restoration and is proved to be useful in many practical cases [41]. For example, SR technique plays an important role in surveillance application where high-resolution images are often required for the purposes of target detection and discrimination.

To obtain a HR image, the basic premise is the availability of multiple LR images captured from the same scene, but at different "looks" [41]. Each LR image is assumed to be a naturally shifted version of other LR frames at subpixel precision. If LR images have different subpixel shifts, then SR is possible as illustrated in Fig. 3.1. If LR images are shifted by integer units and are not subject to other distortions, then each image contains the same information, and SR is not possible.

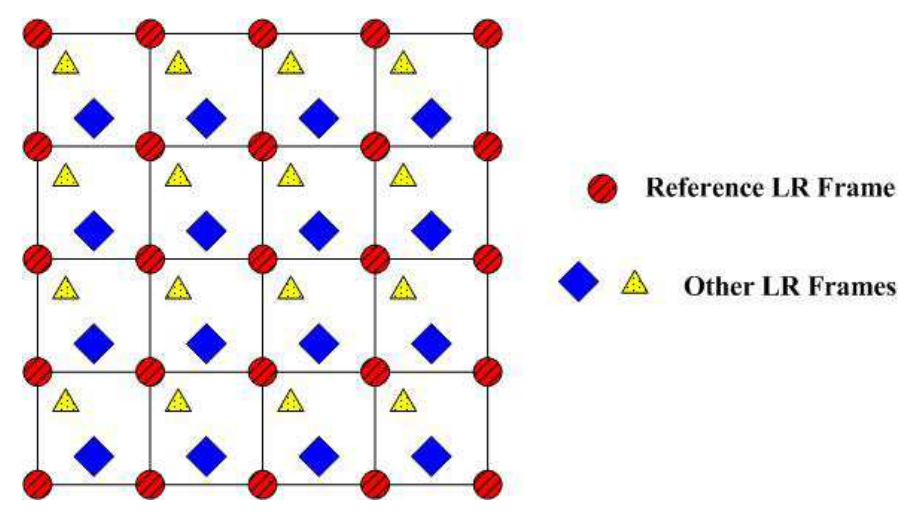

Figure 3.1: Basic Premise for Super Resolution.

The first step to analyze the SR image reconstruction problem is to formulate an observation model. As illustrated in Fig. 3.2, the desired HR image $x$ is warped (includes translation and rotation) to $k$ th warped HR image $x_{k}$, which will further be blurred and down sampled to $k$ th observed LR image $y_{k}$. Assuming that each LR image is corrupted by additive noise, we can then represent the observation model as [41]: 


$$
y_{k}=D B_{k} M_{k} x+n_{k}, \quad \text { for } 1 \leq k \leq p
$$

where $M_{k}$ is a warp matrix, $B_{k}$ represents a blur matrix, D is a subsampling matrix and $n_{k}$ represents a noise vector.

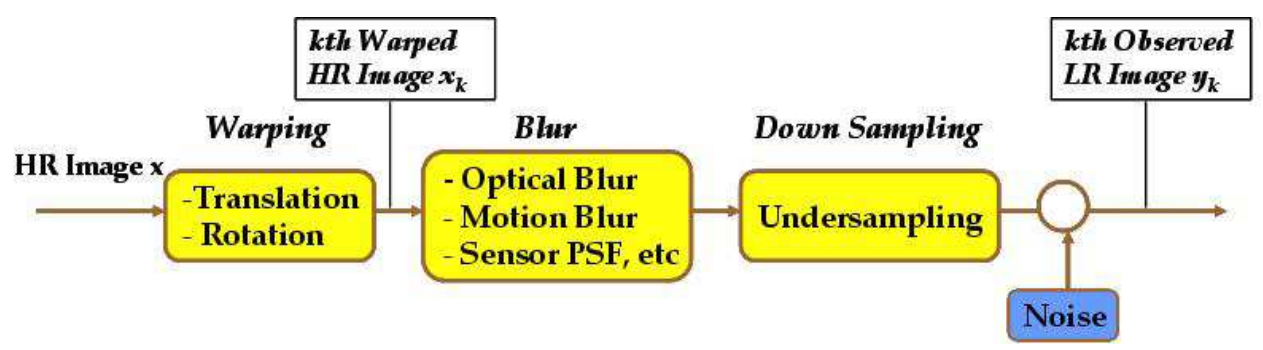

Figure 3.2: Super-Resolution Observation Model.

The literature describes a variety of approaches that exploit SR techniques (for example, [41],[42]). In this thesis, we focus on the interpolation-based approach which is the most intuitive method for SR image reconstruction. The computational load of this kind of approach is low, so it is suitable for real-time application in our UAV-based ATD system. Basically, the approach consists of image registration. A block-diagram of the processing system is presented in Fig. 3.3. Image registration is the process of matching two images so that corresponding coordinate points in the two images correspond to the same physical region of the scene being imaged [43]. After the first stage, the LR images are registered relative to a specific frame of reference. Following this process, available LR pixels are used to sparsely populate a HR image grid, and a non-uniform interpolation techniques are applied to the remaining gridpoints to generate an estimate of the HR image. Finally, debluring and denoising techniques are applied to get a clear image.

\section{Interpolation-based Approach for Improved ATD}

The interpolation-based SR technique implemented in our ATR system is a modified version of the algorithm proposed by Hardie et al. [44] which is recommended for real-time resolution enhancement of data acquired by an infrared sensor. The main 


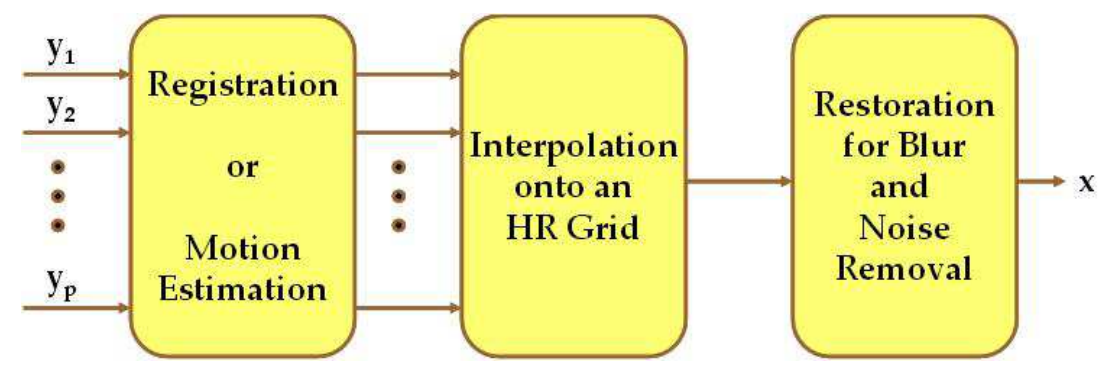

Figure 3.3: A Block-diagram of the Interpolation-based Approach.

reason we adopted such algorithm is due to its high operational speed. The results demonstrating the effect of SR on detection performance are provided in Sec. 4.3.1.

Before reconstructing a HR image, we should align all LR images and project onto a HR grid. In our ATD system, the aerial images are captured by swarmed UAVs from different angles of view. Therefore, the original LR images usually represent relatively large displacements, which require more advanced registration techniques compared to normal super-resolution system. To successfully solve this problem automatically, a two step procedure is proposed. In the first step, we use optical flow [43] to extract similar features in different frames and then apply purely geometric matching procedure (detailed algorithm is in Section 3.1.2) [43]. In the second step, sub-pixel image registration is achieved by a state-of-the-art gradient-based registration technique [44].

After aligning all images and projecting them onto a HR grid, a non-uniform linear interpolation method [45] is used to generate a high-resolution grid. We then use Wiener filter [46] to deblur and get a clear HR image.

\subsubsection{Image Mosaicking for Improved ATD}

Among challenges faced in optical ATD, occluded object detection is a special and important issue. Several ATD/R algorithms, which account for obscuration have appeared in the literature (for instance, [47],[48]). In our swarmed UAV system, an image mosaicking technique could be applied to solve the problem. A more complete image of an object to be detected generated by collecting information from multiple UAVs is expected to improve the ATD performance. Our image mosaicking approach 
consists of two parts: image registration and mosaicking.

We adopt a control-point based image registration algorithm [43] which is suitable for remote sensing imagery. The algorithm proposed by Kenney et al. [43] consists of a two-step procedure: control-point extraction and matching. A FAST feature detector [49] is first employed to extract prominent feature points from two or more images. In the second step, candidate points in one image are matched with candidate points in the other images. A "coarse" control-point matching process is accomplished by comparing features at each control point in the first image with features at each control point in the other images. A purely geometric matching procedure is employed to further refine the matching results.

After the matching of control points is performed, an affine transformation function is estimated using the least squares method. After registration, a simple image mosaicking [43] is applied by equalizing the images in the overlapping area.

\subsection{Score-level Data Fusion for Improved Detec- tion}

\section{Scenarios and Assumptions}

Consider now the case when frames contain unoccluded targets represented by a large number of pixels sufficient for successful detection. The images, however, may be of poor quality, which as we expect degrade the detection performance. Motivated by classifier combination schemes in pattern recognition [50], we design a two-step data fusion procedure at the score-level and demonstrate that this results in improved detection performance (Section 4.3).

When designing a pattern recognition system, it is possible to combine different classifiers to achieve a better classification performance. Rather than rely on a singe decision making scheme, classifier combination use all the designs for decision making by combining their individual opinions to derive a final decision [50]. Various classifier combination schemes have been devised and applied to many pattern recognition problems, such as biometrics [51][52]. 
In a swarmed UAV-based ATD system, images of the same target may be acquired by a single or multiple UAVs at different view angles. These images are of relatively high resolution. This type of data are not easy to describe by a probabilistic model, which leads to an optimal detector design. Therefore, following the ideas of classifier combination in pattern recognition [50], we design a score-level data fusion technique for improved automatic target detection. The fusion procedure is as follows.

First, the frames containing information about the same target are registered with respect to a reference frame using the control-point based image registration method [43]. Then the single-frame object detector described in Chapter 2 is applied to the overlapping areas of both the reference images and registered images. The scores produced by the stage classifiers (2.3) applied to different image frames are combined to generate the final detection results. Kittler et al. [50] summarize on classes of combination strategies at the score level. We choose Average Rule and Majority Vote Rule derived from Sum Decision Rule because of their simplicity and computational efficiency.

The Average Rule is described by the following equation:

$$
C\left(x_{1}\right)_{\text {fused }}=\left\{\begin{array}{cc}
1, & \text { if } \frac{1}{N} \sum_{n=1}^{N} \sum_{j} h_{j}\left(x_{n}\right)>T \\
0, & \text { otherwise }
\end{array}\right.
$$

where $x_{1}$ is the specified reference image frame and $x_{n}$ is a sub-window from image $n$ among the $N(N \geq 2)$ frames of registered images.

The Majority Vote Rule is given by:

$$
\begin{gathered}
C\left(x_{n}\right)=\left\{\begin{array}{cc}
1, & \text { if } \sum_{j} h_{j}\left(x_{n}\right)>T \\
0, & \text { otherwise }
\end{array}\right. \\
C\left(x_{1}\right)_{\text {fused }}=\left\{\begin{array}{cc}
1, & \text { if } \sum_{n} C\left(x_{n}\right)>\frac{N}{2} \\
0, & \text { otherwise, }
\end{array}\right.
\end{gathered}
$$


where $x_{1}$ is the specified reference image frame and $x_{n}$ is a sub-window from image $n$ among the $N(N \geq 3)$ frames of registered images. 


\section{Chapter 4}

\section{Numerical Results}

In this chapter, we describe our experimental setup and present numerical results. The data base for training and testing our detector is described in Section 4.1. Section 4.2 presents a summary of results for a single-frame detector. Section 4.3 demonstrates improved detection performance due to data fusion.

\subsection{Database Description}

This section describes the training and testing datasets for our automatic target detector. Section 4.1.1 describes the generation of the training and original testing datasets for a single-frame detector. In order to evaluate the capabilities of the detector under different environmental and camera effects, we generate a dataset of images by simulated effects. This process is described in Section 4.1.2. Also, we generate testing datasets of occluded targets, which is described in Section 4.1.3. This dataset is used to evaluate performance of the detection algorithm on partially occluded target.

\subsubsection{Simulated Optical Data Set}

An ATR Training Tool provided by Augusta Systems Inc. was used to build a simulated database. The tool is capable of generating prospective projections of 18 distinct 
objects projected at different orientation and elevation angles and sampled at distinct resolutions. The objects can be manually superimposed onto a background to simulate various ground conditions (sand, grass and white background). The camera parameters such as position $(x, y)$, azimuth $\alpha$, declination $\beta$ and distance $d$ can be varied to simulate an UAV flight (Fig. 4.1). The resolution of captured images can be adjusted from $512 \times 384$ to $1152 \times 864$. A snapshot of the Graphical User Interface (GUI) of the tool is shown in Fig. 4.2.
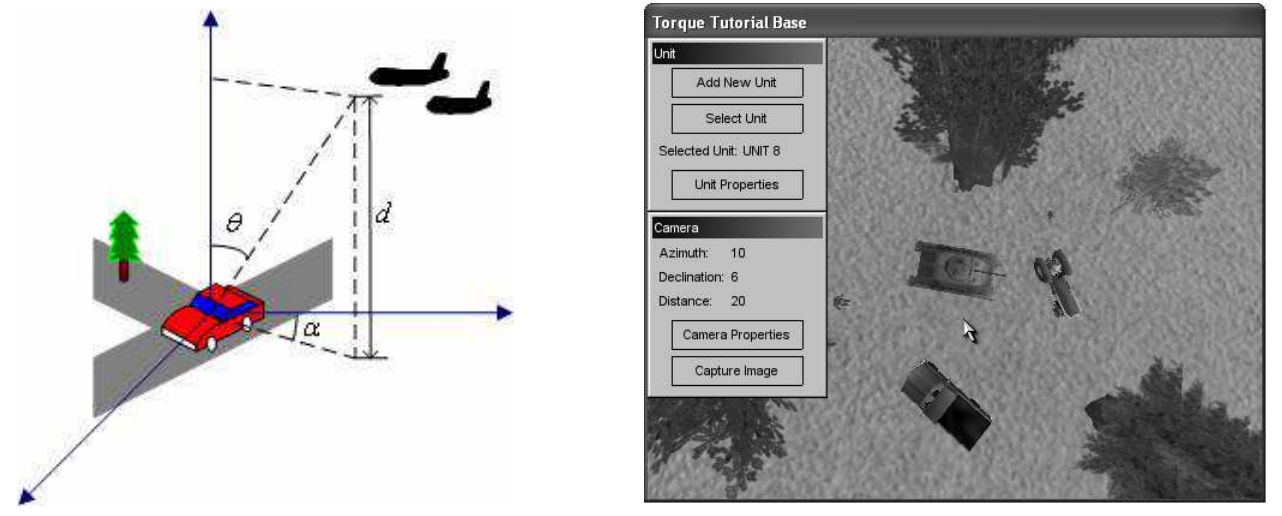

Figure 4.1: Model for Capturing Im- Figure 4.2: The GUI of the ATR trainages. ing tool.

In our ATD system, we adopt the modified Viola-Jones object detector described in Chapter 2 as our single-frame target detector. We choose the tank in the ATR tool as our object of interest (target). To train the detector, a set of target and non-target training images are used. The target training set consisted of 180 cropped targets scaled and aligned to a base resolution of 50 by 50 pixels. The targets are captured by the ATR Training Tool using various camera parameters. The camera parameters cover 360 degree azimuth angle spaced 5 degree apart and 60 degree declination angle spaced 15 degree apart. The non-target subwindows used to train the detector come from 500 images which are captured by the ATR Training Tool. Some typical target and non-target examples are shown in Fig. 4.3 and Fig. 4.4.

In our evaluations of detection capabilities of our single-frame detector we use a testing dataset consisting of 277 gray-scale images generated using ATR training tool. These images contain 440 targets parameterized by varying type, location, 


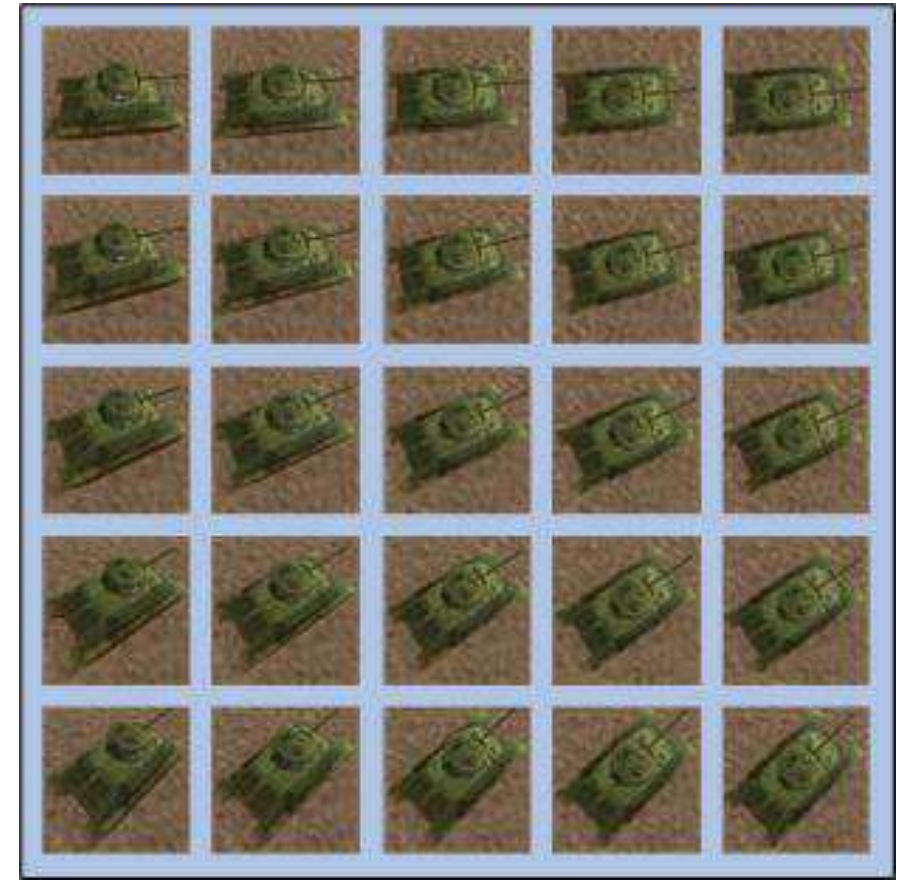

Figure 4.3: Example of Target Images Used for Training.

orientation, and camera elevation angle. Some typical testing examples are shown in Fig. 4.5.

\subsubsection{Simulated Environmental and Camera Distortions}

In the considered UAV network, the fidelity of data gathered by CCD cameras is limited by the quality of the optical and electronic components of the system. Such images suffer from blurring effects and noise from optical and electronic sources.

In order to evaluate detector performance under different environmental and camera effects, we generate additional distorted data. Each original testing image is distorted by adding five different simulated effects, resulting in five additional testing images. Each image contains distortion of one type. We do not combine distortions. The distorted images include one of the following factors: Gaussian noise, illumination effect, varying contrast, motion blur and defocus blur. By controlling the value of the parameters, different levels of distortions in images can be generated. All distortions are generated at five levels, for Level 0, no distortions, through Level 4, maximum 

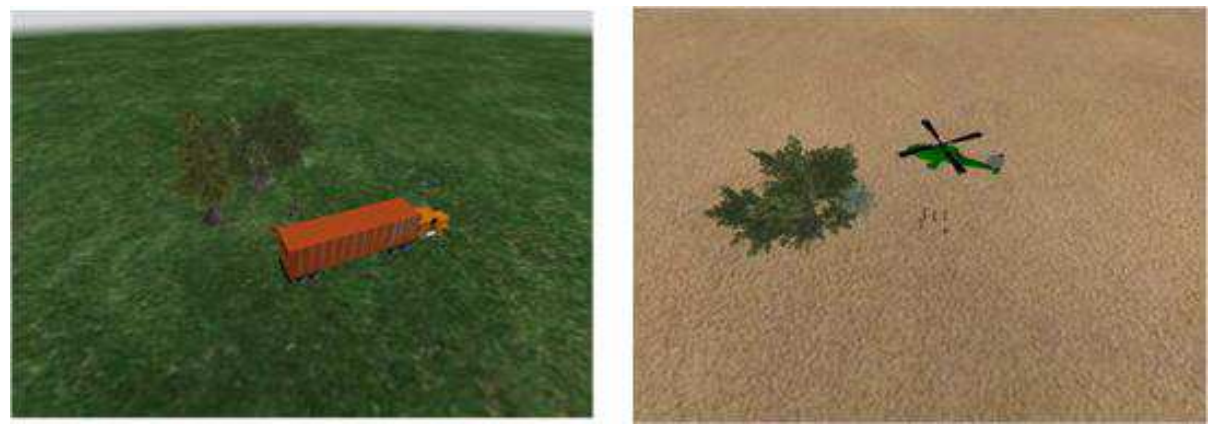

Figure 4.4: Example of Non-target Images Used for Training.
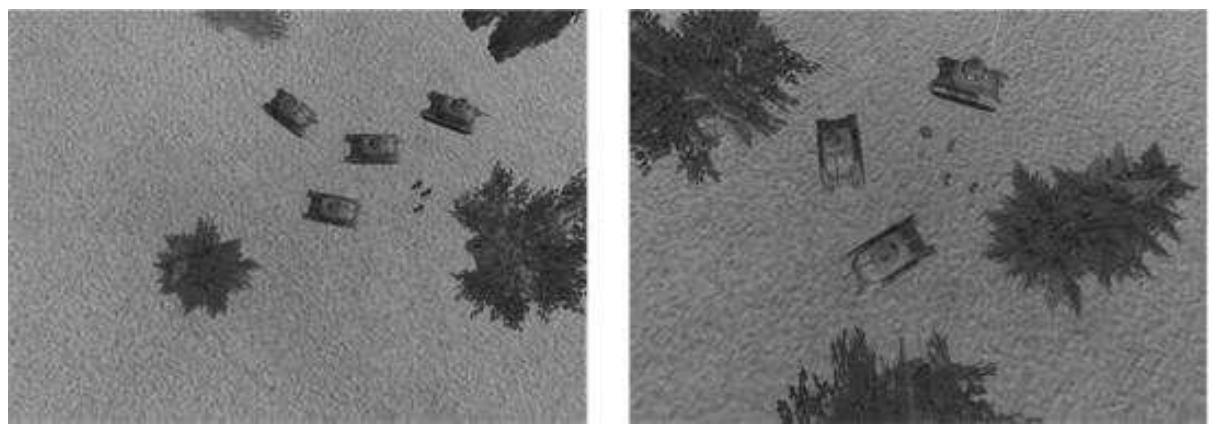

Figure 4.5: Example of Testing Images.

distortions. The details of generation procedures are summarized below.

1. Images contaminated by Gaussian noise contain additive white noise with zero mean and variance $\sigma^{2}$. The variance $\sigma^{2}$ takes values in the range from 0.005 to 0.02 spaced 0.005 apart for Level 1 to Level 4, respectively.

2. The images are brightened or darkened by increasing or decreasing the intensities. This procedure simulates illumination effect. Denote by $\beta$ the parameter that controls the level of illumination. We first normalize image intensities to $(0,1)$, then brighten images by raising to the power of a number less than one, that is, $(1-\beta, \beta \in(0,1))$ or darken images by raising to the power of a number larger than one, that is, $\left(\frac{1}{\beta+1}, \beta \in(-1,0)\right)$. The parameter $\beta$ is set to be -0.8 and -0.4 at Levels 1 and Level 2 for dark images and is set to be 0.4 and 0.8 at Levels 3 and 4 for brightening images.

3. We model contrast change by linear mapping the normalized histogram to a new 
one. If the histogram is "squeezed," then the new image will have low contrast. The more compression, the lower the contrast is. The range is determined by parameter $1-2 T O L$ with TOL taking values in the range from 0.15 to 0.35 spaced 0.05 for Level 1 to Level 4, respectively.

4. A linear relative motion of an optical camera or an object is simulated by convolving images with a two parameters point spread function (PSF)[53]. Length $L$ in pixels and angle $\theta$ in degrees correspond to motion in specific direction with predefined camera velocity. $L$ takes values in the range from 2 to 8,2 units apart for Levels 1 through 4, respectively. The parameter $\theta$ follows uniform distribution on $\left[0,360^{\circ}\right]$ for all levels.

5. The images are filtered by a two-dimensional circular averaging filter to generate defocus blur[53]. Defocus level corresponds to the radius $r$ of the averaging filter. $r$ takes values from 2 to 8 with the step 2 units for Levels 1 through 4 , respectively.

The samples of distorted testing images are displayed in Fig. 4.6.

\subsubsection{Data for Testing the Effect of Occlusion}

One of the critical problems of object detection is that the detector should be able to handle partial occlusion of object, and spurious or noisy data [54][55]. In order to evaluate detector performance on partially occluded objects, we generate a dataset of occluded objects.

We consider a number of occlusion scenarios. In the first scenario, a single large obscuring block is placed over a portion of a target. This simulates occlusion due to a roof or partial man-made coverage. In the second scenario, clutter is in the term of small blobs randomly scattered over the scene. The number and size of blobs vary. In the third scenario, targets are occluded by 2-D projections of trees and shreds. The blocks and silhouettes of trees are intended to explore some of the aspects of structured clutter. The details of generation procedures are summarized below. 
1. In the single block scenario, the clutter takes the form of a large obscuring block placed on objects which occludes a portion of the whole object. The intensity of the pixels within the block is the same as the intensity of the background. The width of the block is varied to generate different level of occlusion. The level of occlusion is measured as a ratio of occluded pixels on the target to the total number pixels within the target. Some typical examples are shown in Fig. 4.7 .

2. In the case of blobs, small occluding blobs are randomly placed over the object areas. Two parameters are involved in generation of the occlusion: the total number $n$ of clutter pixels, and the width $w$ of the blobs. These two parameters are related. The total number of occluded pixels on the target is kept constant at each occlusion level. Thus if we increase the size of the blobs, we have to simultaneously decrease the number of blobs. The smallest pixel-size blobs are essentially i.i.d. noise; larger blocks represent increasing amounts of structure.

In placing a blob, its location is selected randomly. We require that blobs do not overlap. This ensures that the total number of cluttered pixels stays constant as the blob size is varied. Some typical examples are shown in Fig. 4.8.

3. In the third scenario, 2-D silhouettes of trees are randomly superimposed onto the scene. The trees silhouettes are formed by thresholding imagery captured by ATR Training Tool. Two parameters are varied: the shape of the tree and the number of trees. The tree models used are shown in the left column of Fig. 4.9. Columns 2 and 3 of Fig. 4.9 show examples of occlusions due to trees. The middle shows the case of a single tree, the right column shows the case of three occluding trees. 


\subsection{Results: Single-frame Detector}

\subsubsection{Learning Results of Single-frame Detector}

The training database for our single-frame detector has been described in Section 4.1.1. A 10 stages cascade classifier with 33 weak classifiers is trained as our singleframe detector. It is much simpler than face detector which has 24 stages with 2913 weak classifiers [4]. We summarize the detail parameters in the Table 4.1.

The first four stages of Haar-like features selected by AdaBoost are visualized in Fig. 4.10. The feature visualization (black and white boxes) is analogous to Chapter 2.1. According to the figure the most important features in single-frame detector seem to be its edge information.

Table 4.1: Training Parameters of Single-frame Detector

\begin{tabular}{|c|c|}
\hline Number of Positive Samples & 180 \\
\hline Number of Negative Samples & 500 \\
\hline Stages & 10 \\
\hline Width $\times$ Height/Pixels & $50 \times 50$ \\
\hline
\end{tabular}

\subsubsection{Influence of Environmental and Camera Effects on De- tection Performance}

In our evaluations of detection capabilities of Haar feature-based algorithm we use a dataset consisting of 277 grayscale images generated using the ATR training tool which is described in Section 4.1.1. These images contain 440 targets parameterized by varying type, location, orientation, and camera elevation angle. We further generate 5 distorted images per each original "clean" image. The distorted images include illumination variations, contrast variations, Gaussian noise, defocus blur and motion blur which are described in Section 4.1.2. The effects are generated individually and tested separately. For each effect, the distortion is increased from low level to high 
level in which level 0 corresponds to the case when no distortion is imposed. The results of detection performance evaluation are shown in Fig. 4.11(a)-(e).

From the ROC curves, we can conclude that illumination and contrast variations do not affect the detection performance significantly. This is because the Haar featurebased detector implements a light correction procedure prior stage classification. To be more specific, prior to stage classification all test windows are normalized to minimize the effect of different lighting conditions. The procedure of normalization is as follows:

$$
I^{-}(x, y)=\frac{I(x, y)-\mu}{c \sigma}, c \in R^{+}
$$

where $I(x, y)$ is the pixel value within the sub-window during detection scanning. $\mu$ and $\sigma$ are the mean and the standard deviation of $I(x, y)$.

Of the effects, both blur and noise degrade detection performance: the number of false alarms in detection increases with increased level of effects.

\subsubsection{Influence of Occlusion on Detection Performance}

Three experiments are conducted to evaluate the performances of the Haar-like object detector on partially occluded objects. In the first experiment, targets are occluded by a single block. In the second experiment, randomly positioned blobs are used to occlude portions of a target. In the last experiment, the occlusion are due to trees. The testing databases were described in Section 4.1.3. The results of detection performance evaluation are shown in Fig. 4.12.

In the single block scenario, the width of the block is varied to generate 5 ROC curves shown in Fig. 4.12(a). The results demonstrate substantial loss in performance when a generated single block obscures more than $50 \%$ of a target.

The results of the experiments involving multiple blobs are shown in Fig. 4.12(b). Again, in this experiment, the total number of cluttered pixels is fixed. Note that the performance degrades as the block size increases. In essence, the low-correlated clutter has comparatively little effect on the algorithm. The algorithm becomes more confused when the clutters (blobs) increase in size to add significant features to or remove significant features from the targets. 
The results of the third experiment of trees scenarios are shown in the Fig. 4.12(c)(e). Clearly, performance degrades as the number of trees increases. Also notice Tree 1 and 2, which have a broader, spread-out appearance, induce worse performance compared to Tree 3 which has a solid, concentrated appearance.

\subsection{Results: Multiple-frame Detector}

To evaluate improvement in detection performance due to involvement of multiple frames, we consider three fusion scenarios: fusion at image level (Super-resolution and image mosaicking) and fusion at the score-level. The first scenario will be beneficial in the case of low resolution images. The second scenario may improve the performance of the detector when partially occluded targets are present in a scene. And the last scenario results in performance improvement under a broad range of conditions.

\subsubsection{Detection Performance: Super-Resolution for Improved ATD}

We use an interpolation-based super-resolution technique as a method of fusing data at the image level. To generate the high resolution (HR) image, the low resolution (LR) images are registered relative to a specific frame of reference by a two-step registration procedure. In the first step, we use optical flow to extract similar features as control points in different frames and then apply a matching procedure (details of the algorithm were provided in Section 3.1.2) [43]. In the second step, sub-pixel image registration is achieved by a gradient-based registration technique [44]. Following registration, available LR pixels are used to sparsely populate a HR image grid, and non-uniform interpolation techniques are applied to the remaining gridpoints to generate an estimate of the HR image.

To demonstrate abilities of the selected super-resolution technique, we use a sequence of four frames of the same area taken by a UAV flying the area. The UAV flies at the attitude of 750 meters. The images were taken 1.5 seconds apart. We assume the white round building is the target of interest. The original LR images have the 
size of $256 \times 256$ pixels and are shown in Fig. 4.13 (a). The circle points are the control points selected for image registration. After the first-step coarse registration, the LR images are partitioned into the overlapping areas $(235 \times 126)$ shown in Fig. 4.13 (b). The final high resolution image of size $470 \times 252$ is shown in Fig. 4.13 (c). The resulted HR image gives us a more detailed information about the target, which is beneficial for performing detection task.

To evaluate improvement on detection performance due to SR technique, we conduct an experiment on synthesized image databases. High-resolution (HR) images are constructed from artificially-generated, low-resolution (LR) images. We train two detectors on the same set of positive images but at different image resolutions. The high-resolution detector is trained on positive samples of $30 \times 30$ pixels. The low-resolution detector is trained on positive samples of $15 \times 15$ pixels. The size of positive samples is determined by the minimum size of targets in images submitted for detection. The summary of parameters is provided in Table 4.2.

Table 4.2: Summary on High Resolution and Low Resolution Detectors used in our experiments

\begin{tabular}{|c|c|c|}
\hline & LR Detector & HR Detector \\
\hline Number of Postive Samples & 180 & 180 \\
\hline Number of Negative Samples & 500 & 500 \\
\hline Stages & 11 & 13 \\
\hline Width $\times$ Height/Pixels & $15 \times 15$ & $30 \times 30$ \\
\hline
\end{tabular}

The two detectors were tested using three datasets: low-resolution(LR), highresolution(HR) and super-resolved(SR) test datasets. LR images are generated by randomly translating and rotating images in HR dataset and then downsampling by a factor of 2 in each dimension. SR images are generated using $K$ LR frames. The performance of the detector trained on HR images is tested on a number of SR datasets constructed from $K=2,4$ and 8 frames of LR images. In the ideal case of perfect half-pixel displacement, one needs only 4 LR images to obtain a HR estimate. However, since displacements are random (both translations and rotations), a larger number of LR images is needed for accurate reconstruction. 
The testing results are shown in Fig. 4.14. The performance of LR detector on LR test database is poor. This confirms that LR images lack important details for successful detection. In this case, HR detector considerably outperforms SR detector.

\subsubsection{Detection Performance: Image Mosaicking for Improved ATD}

We use an image mosaicking technique as a method of fusing data at the image level which are described in Section 3.1.2. To demonstrate capabilities of the selected mosaicking algorithm, we involve two images acquired by a UAV at the altitude 550 meters. The images are taken 2 seconds apart. The two frames are shown in Fig. 4.15 (a) and (b). The mosaicking results are shown in Fig. 4.15 (c).

\subsubsection{Detection Performance: Score-level Data Fusion}

For score-level data fusion, we employ Average Rule (3.2) to perform data fusion. Two image sequences of the same scene containing several targets are captured from different view angles and distances by the ATR Training Tool to simulate surveillance tasks performed by two independent UAVs. Each sequence consists of 40 frames of images and 160 targets. Some examples of typical frames are shown in Fig. 4.16. The red rectangles indicate detection results. The first row shows detection results before fusion on reference frames captured by UAV 1. The second row shows detection results before fusion on assisting frames captured by UAV 2. The last row show detection results after score-level fusion on reference frames. The ROC curve is shown in Fig. 4.17. The experiments confirm that the score-level data fusion technique can reduce false alarms and keep high detection rate. 


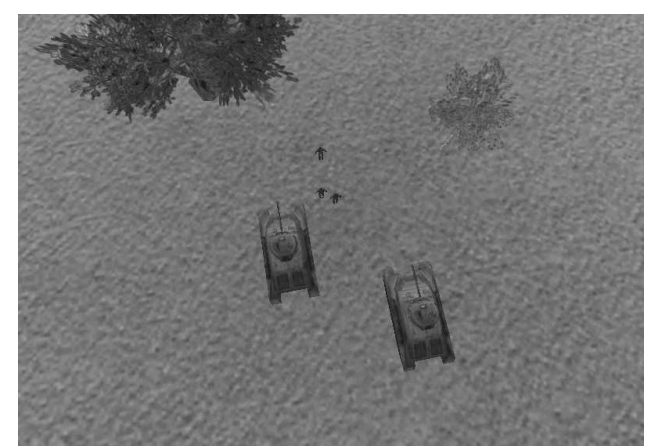

(a) Original

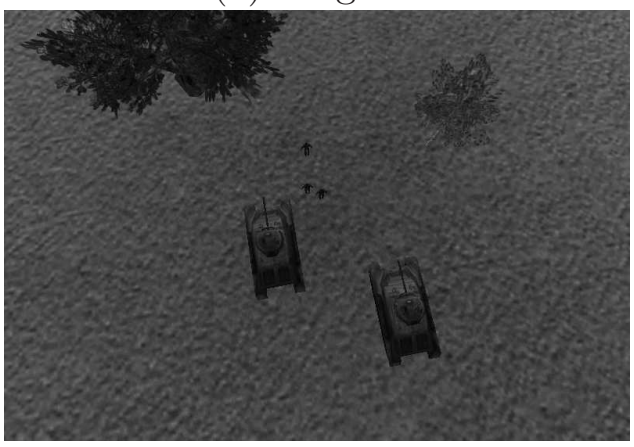

(c) Lighting

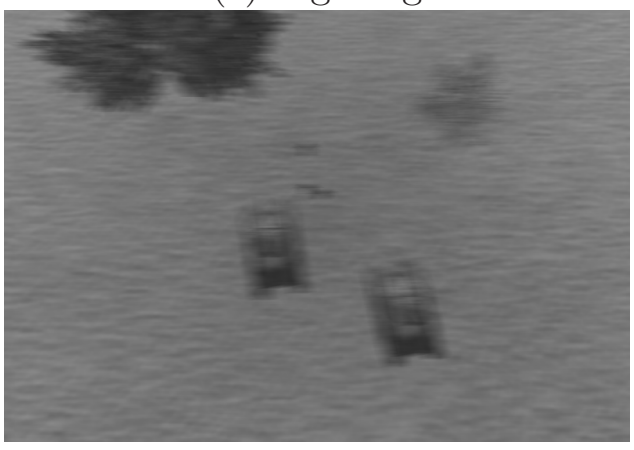

(e) Motion Blur

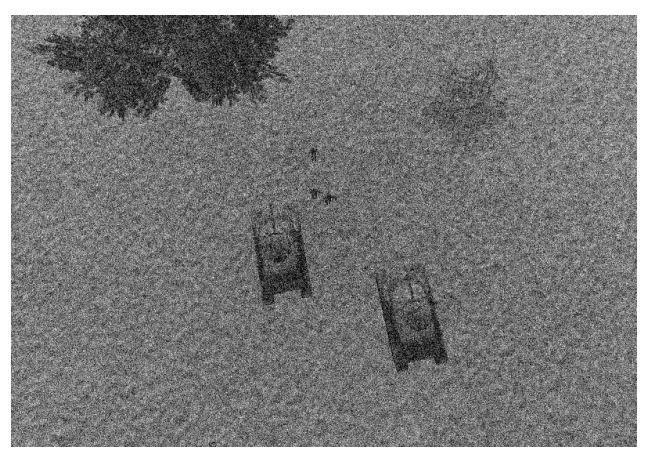

(b) Gaussian Noise

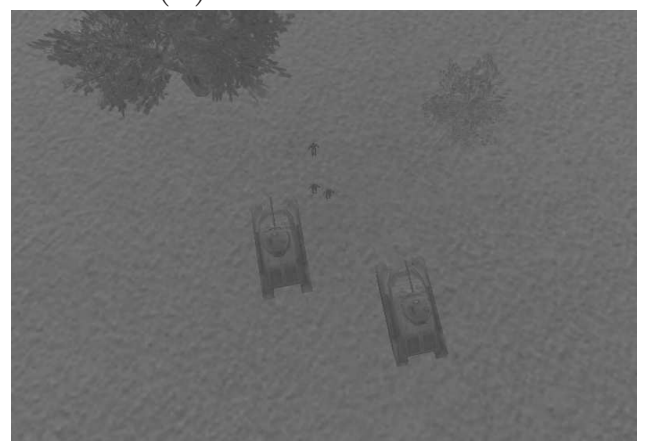

(d) Contrast

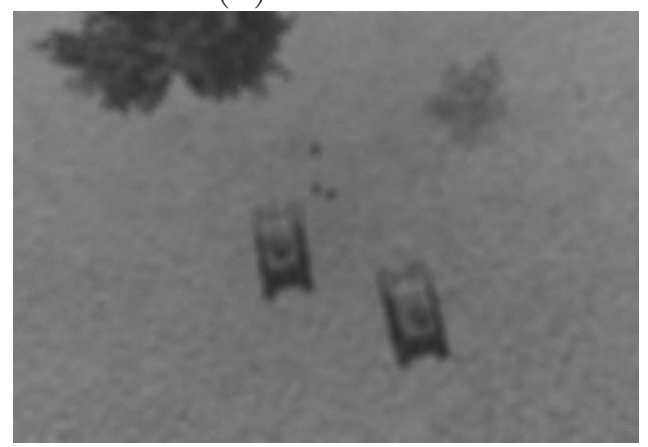

(d) Off Focus Blur

Figure 4.6: Examples of Distorted Testing Images. 


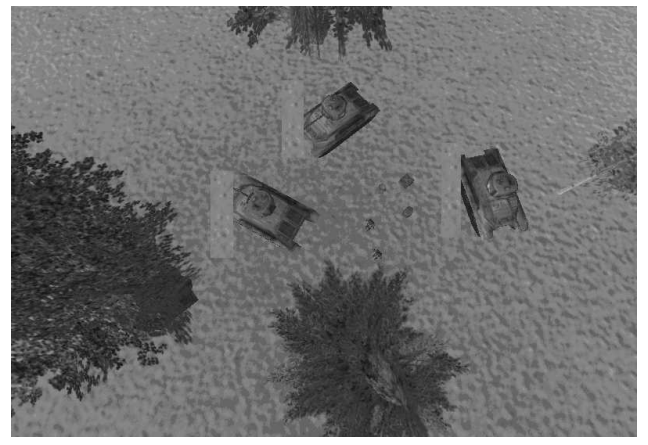

(a) $p=0.2$

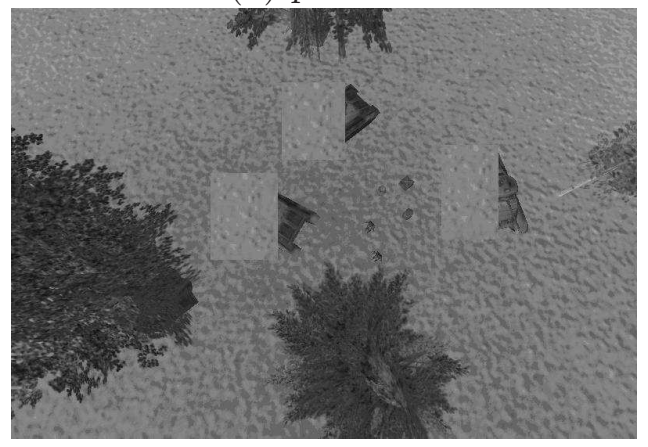

(c) $p=0.4$

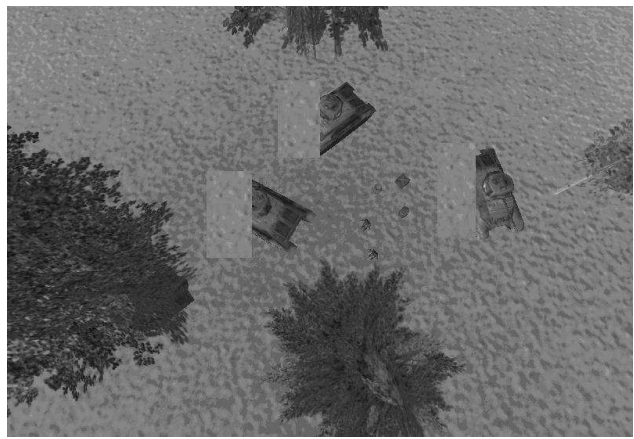

(b) $p=0.4$

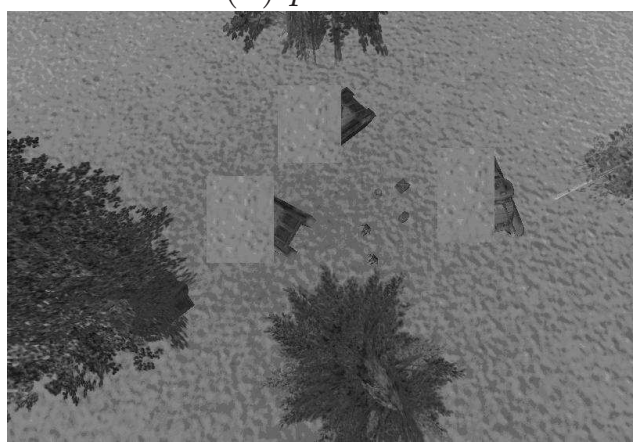

(d) $p=0.6$

Figure 4.7: Examples of Targets Occluded by a Single Block. 


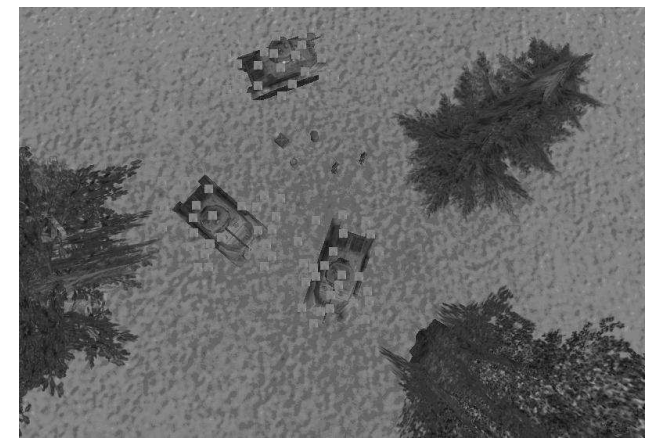

(a) $w=10$

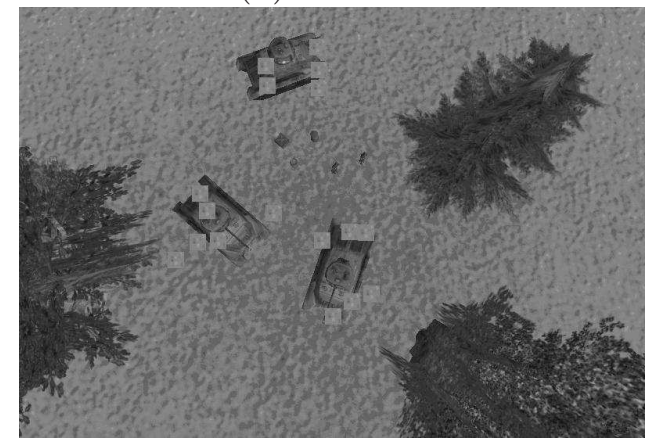

(c) $w=20$

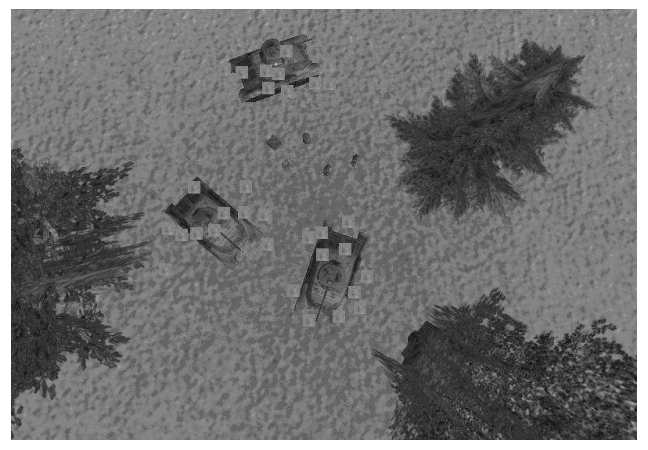

(b) $w=15$

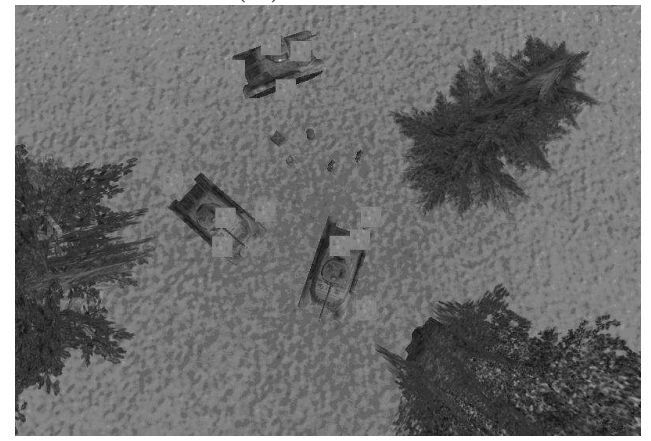

(d) $w=25$

Figure 4.8: Examples of Targets Occluded by Multiple Blocks. 

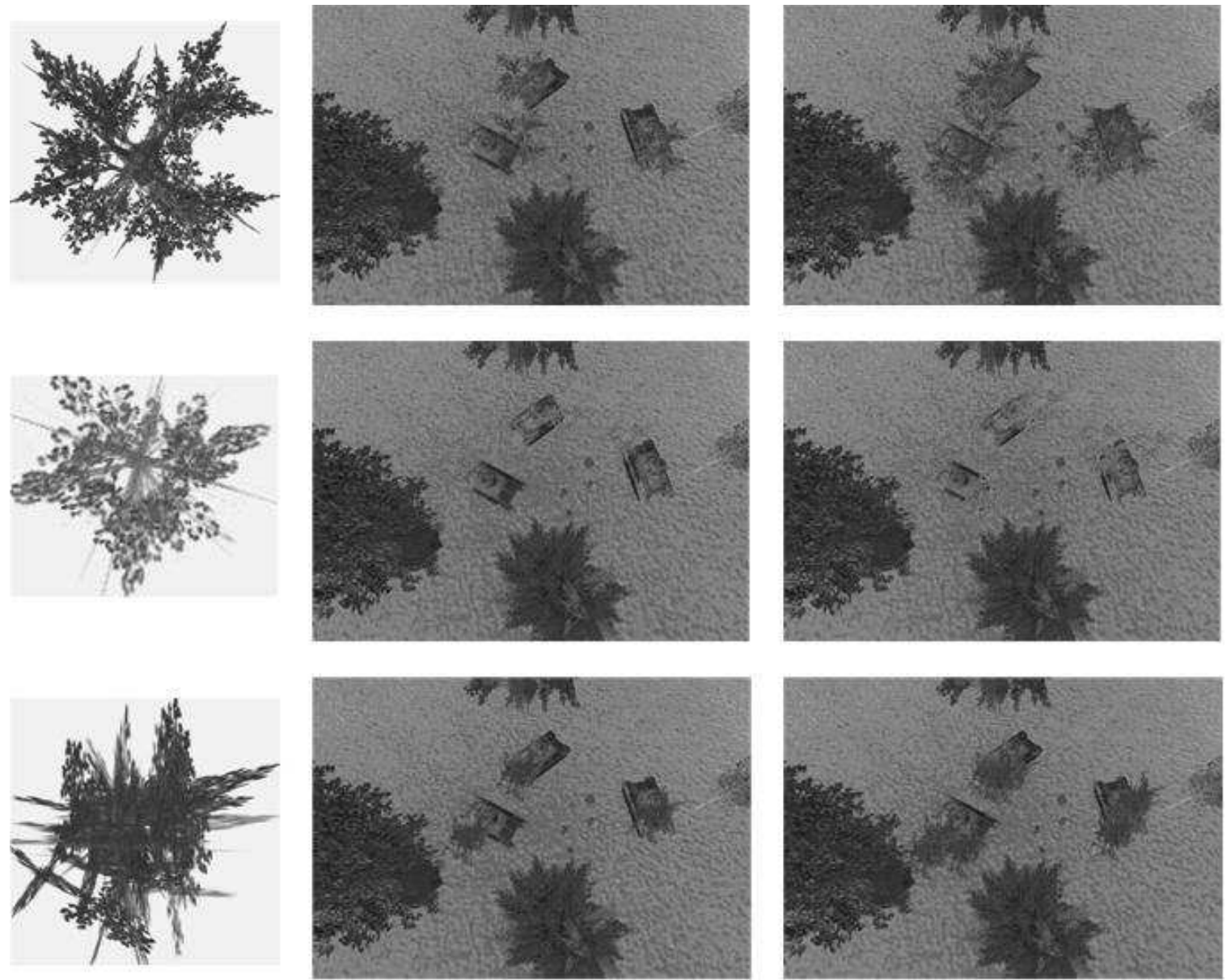

Figure 4.9: Examples of Targets Occluded by Trees.

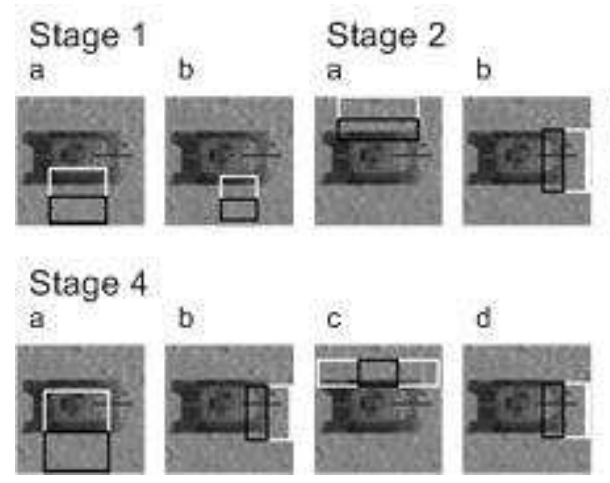

Figure 4.10: Single-frame Detector Features Selected by AdaBoost (First Four Stages). 


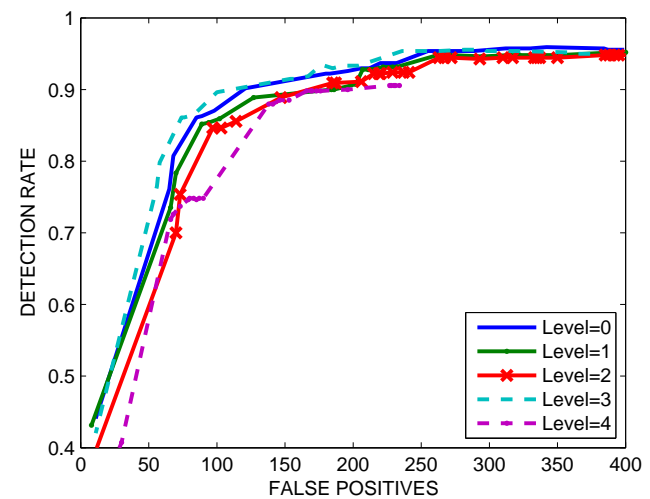

(a) Illumination

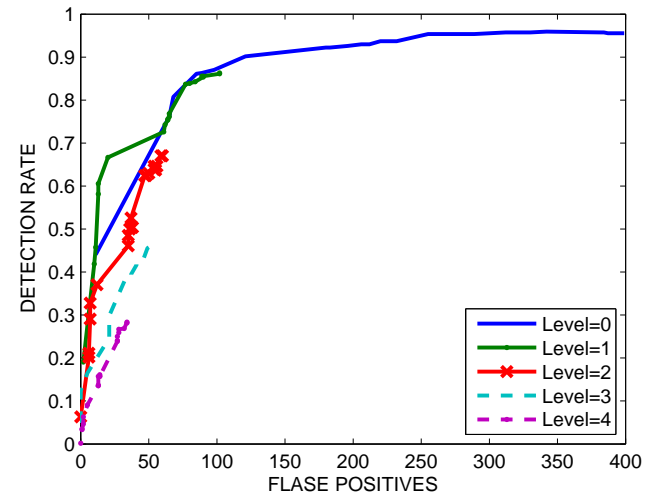

(c) Gaussian Noise

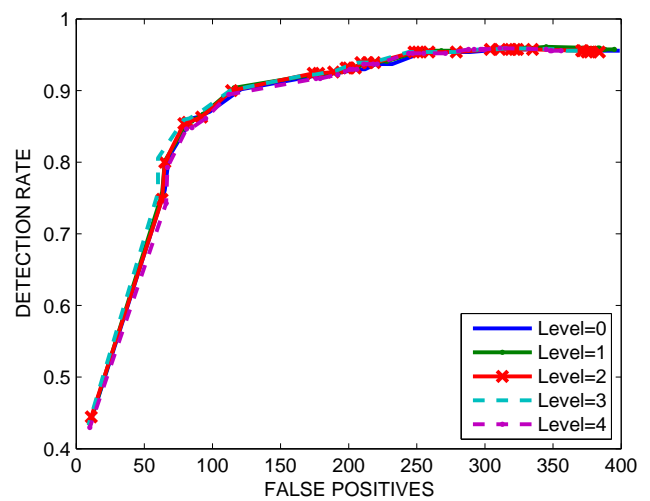

(b) Contrast

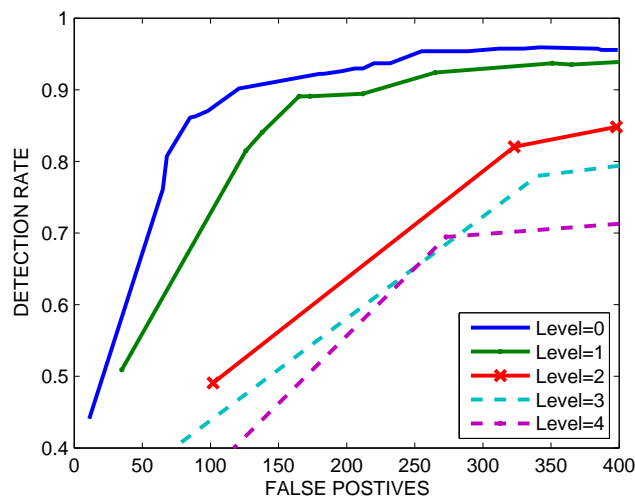

(d) Defocused Blur

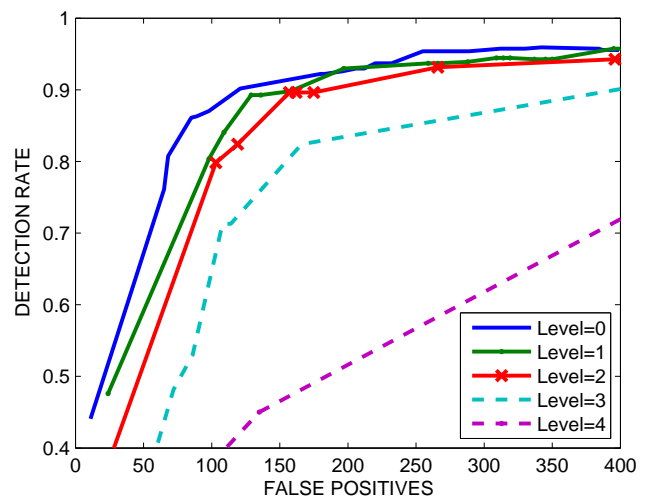

(e) Motion Blur

Figure 4.11: (a)-(e) Detection performance as functions of various environmental and camera effects. 


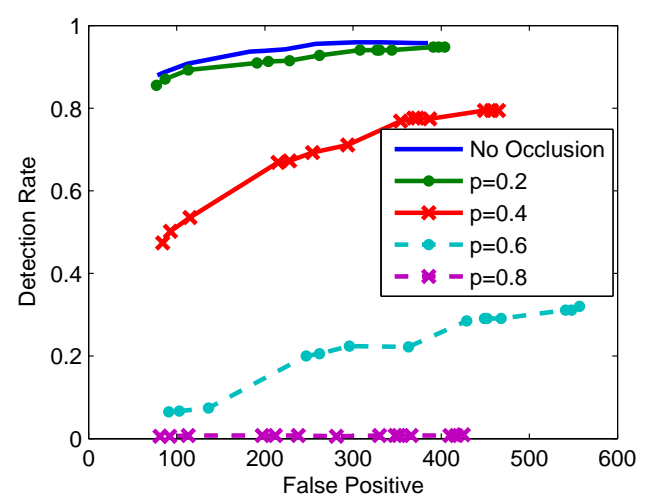

(a) Single Block

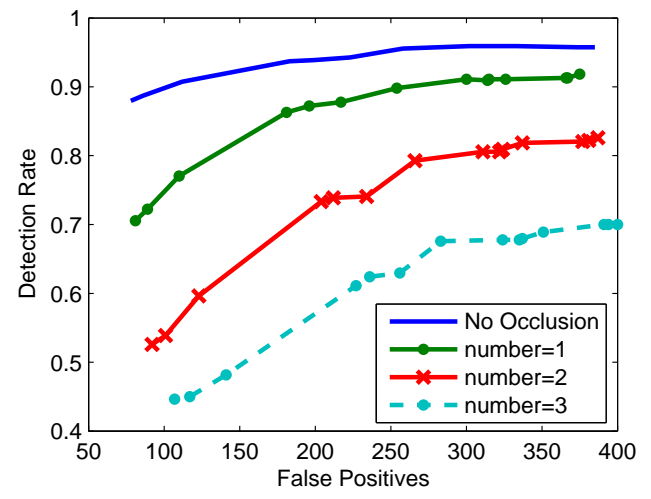

(c) Tree 1

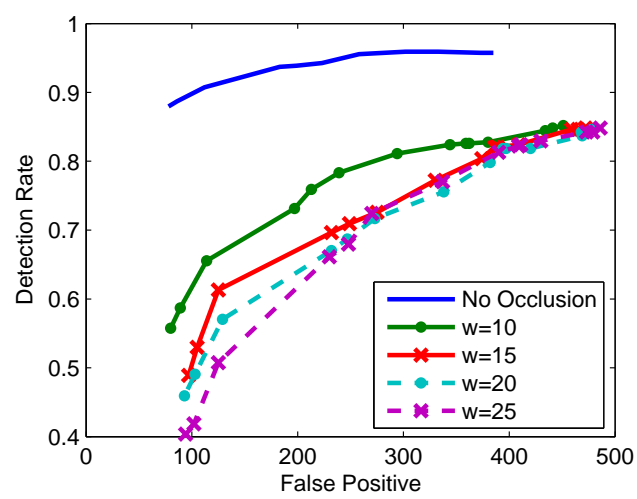

(b) Multiple Blocks

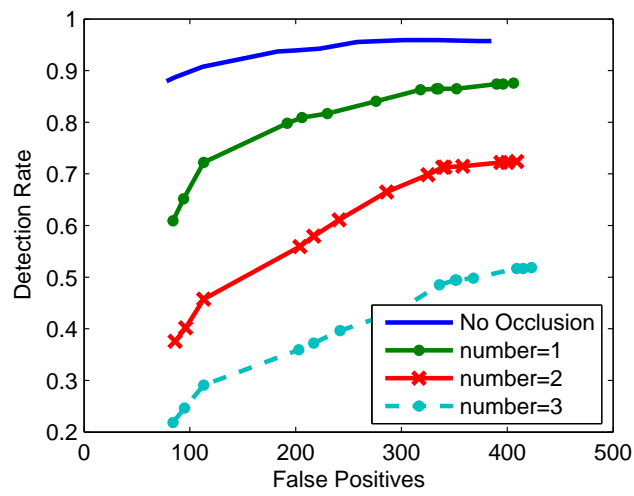

(d) Tree 2

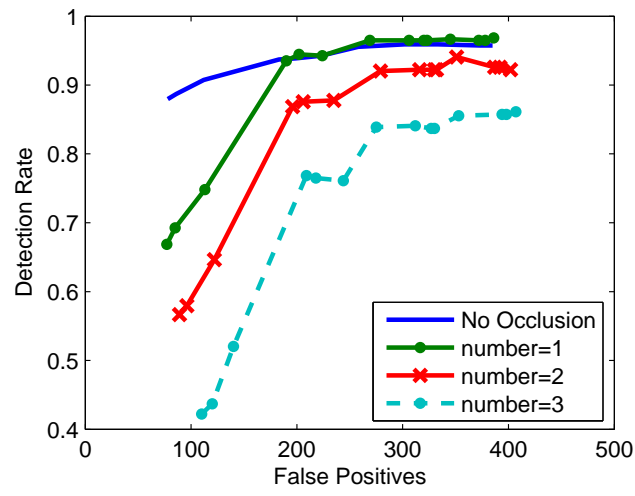

(e) Tree 3

Figure 4.12: (a)-(e) Performance of Occluded Target Detection. 

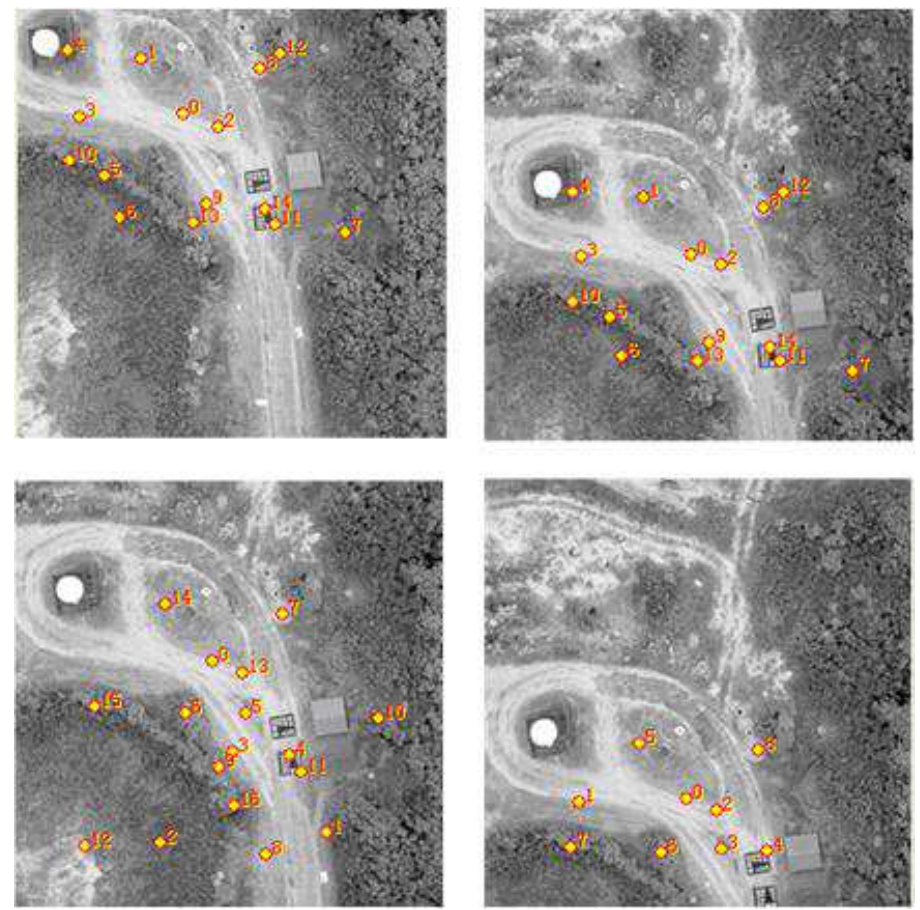

(a) LR Natural Images with Large Displacements $(256 * 256)$

Circle points indicate corresponding control points
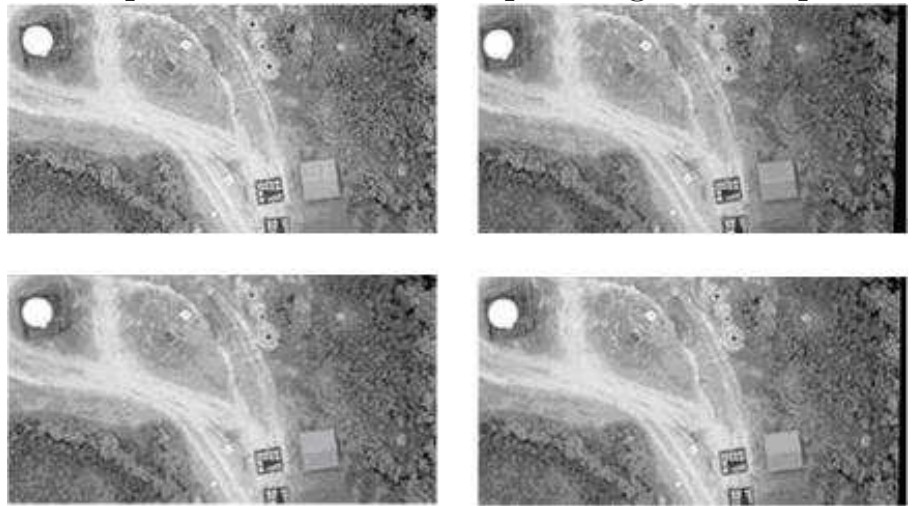

(b) LR Natural Images after First-Step Registration (235* 126)

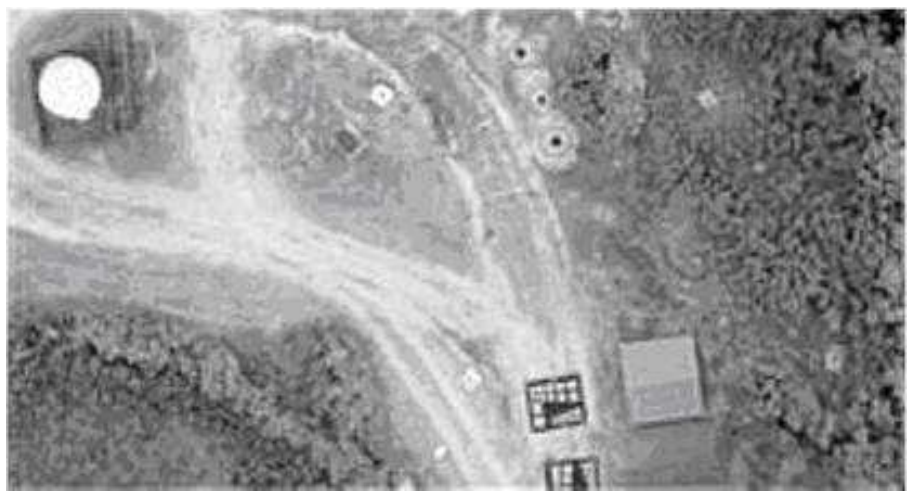

(c) Super-resolved HR estimate using four LR images $(470 \times 252)$

Figure 4.13: Example for Super-resolved Natural Image. 


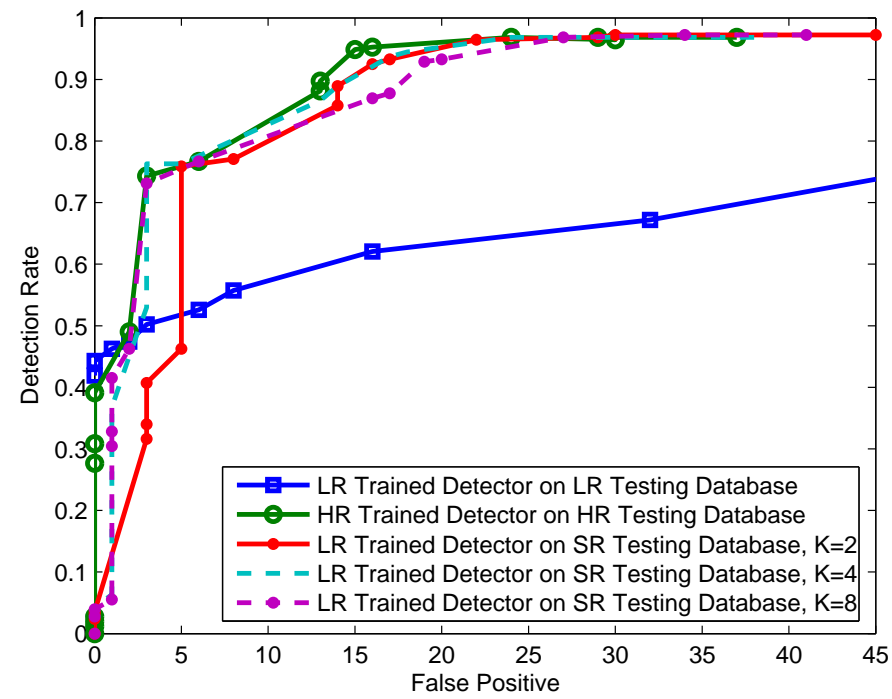

Figure 4.14: Image-level Data Fusion Results. 


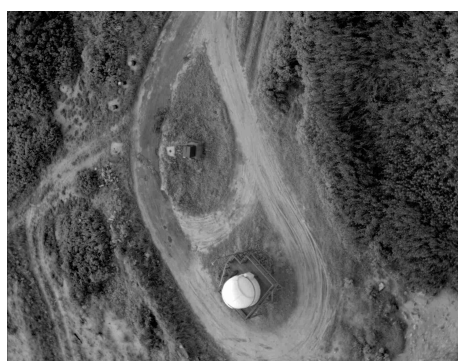

(a1)

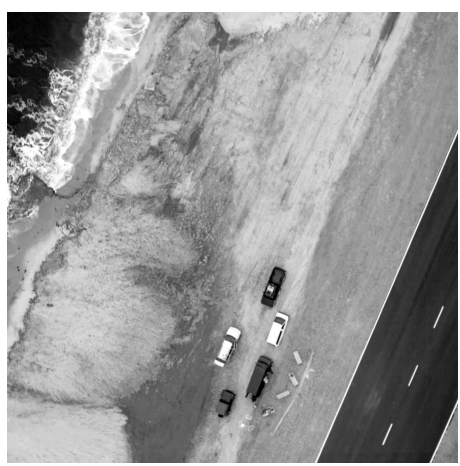

(a2)

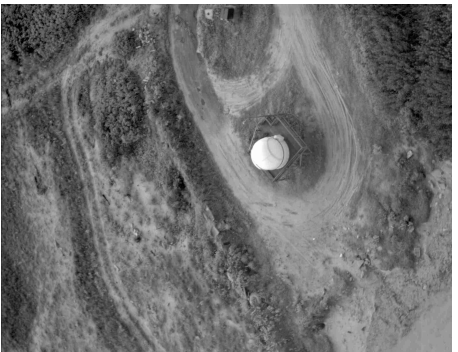

(b1)

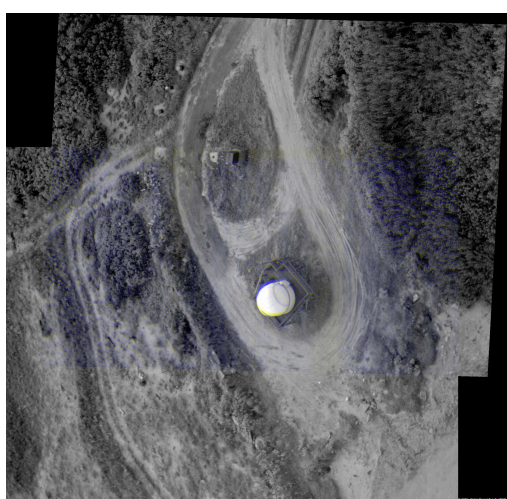

$(\mathrm{c} 1)$

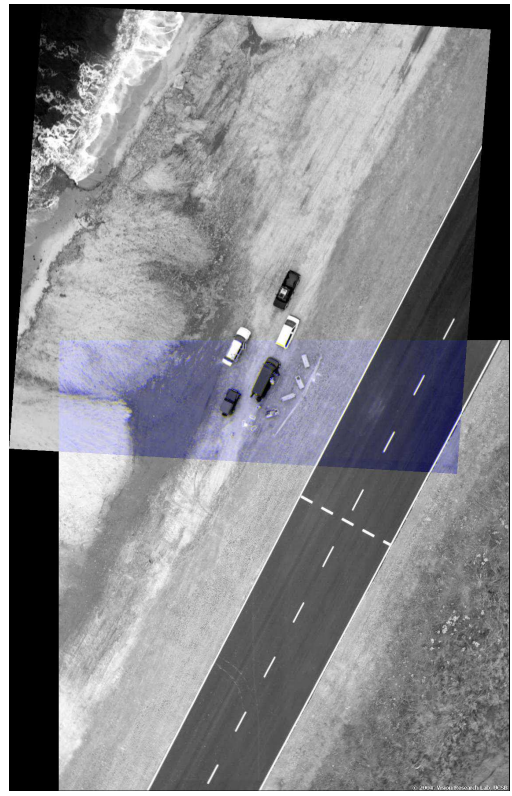

(c2)

Figure 4.15: Example for Image Mosaicking. 


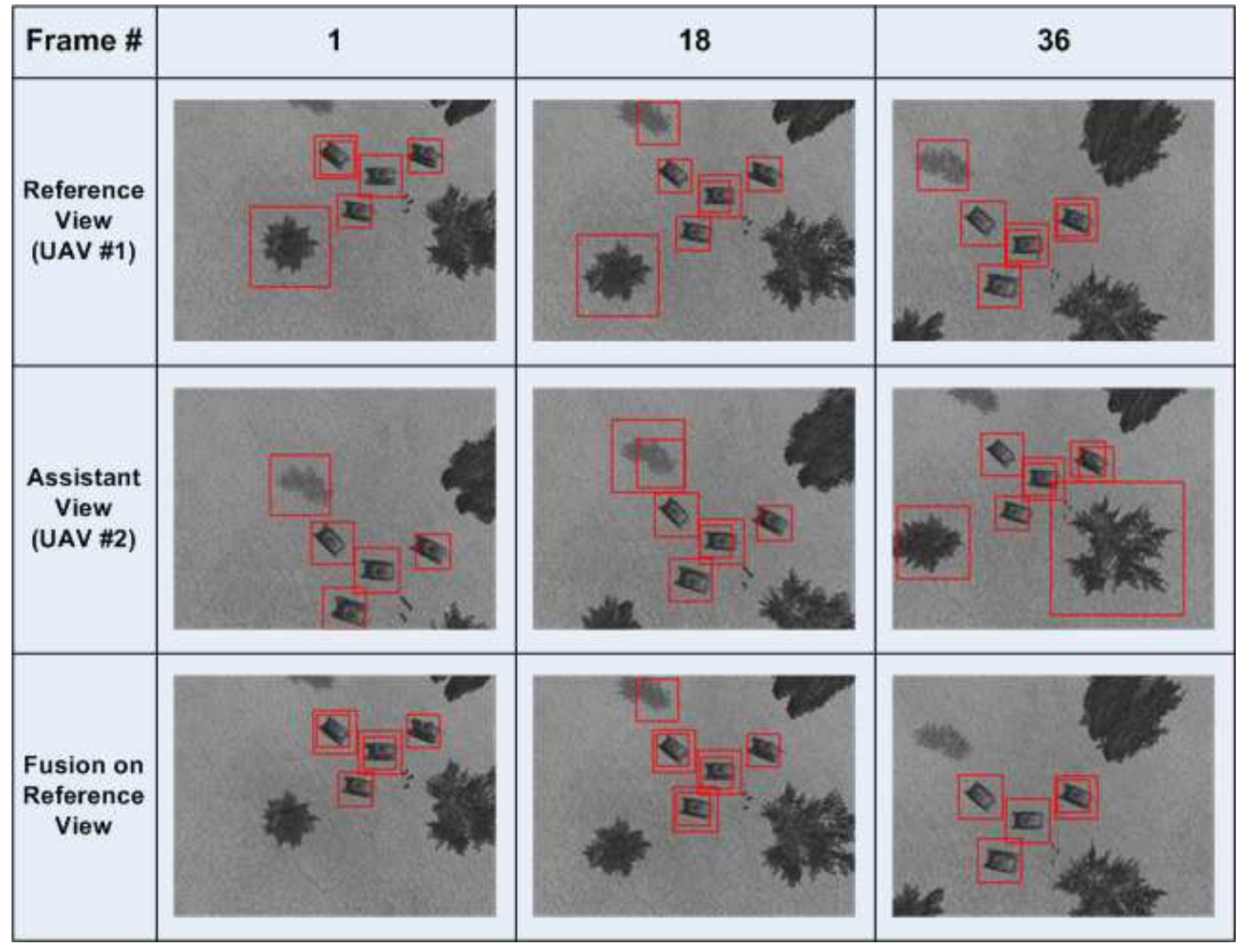

Figure 4.16: Example Frames for Score-level Data Fusion.

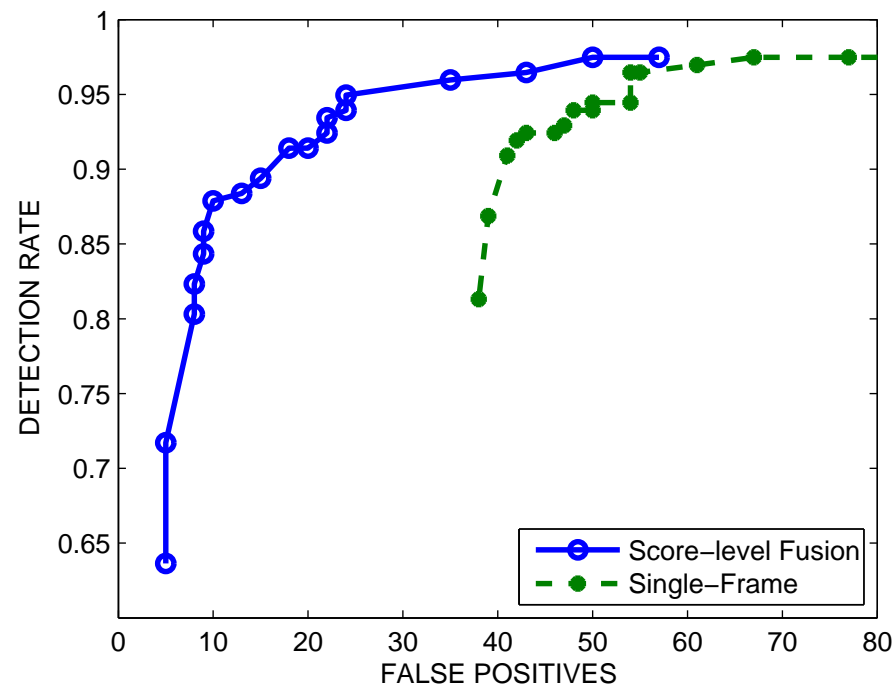

Figure 4.17: Score-level Data Fusion Results. 


\section{Chapter 5}

\section{Conclusion and Future Work}

In this work we advanced the state of the art in automatic target detection in the following ways. We performed a comprehensive research of currently available ATD algorithms from optical imagery. The adopted detector is a modified version of ViolaJones face detector which is a cascade classifier based on Haar-like features. In order to improve detection performance in a swarmed UAV network, we proposed and analyzed several data fusion algorithms at different levels.

Firstly, following Viola-Jones face detector, we trained our automatic target detector to operate on single image. In our system, we focus only on optical images captured by optical cameras mounted on board of UAVs. Such images suffer from sensor limitations, environmental and camera effects. To test the robustness of the detector with respect to non-ideal imagery, we synthesized datasets distorted by individual weather and camera effects. The effects include Gaussian noise, lighting, contrast, motion blur, off-focus blur and occlusion. Degradations of the detector performances due to these effects were further evaluated.

In the second part of the work, we proposed several data fusion methods for improved detection performance. In the first scenario, a super-resolution technique was employed for low-resolution image data. In the second scenario, an image mosaicking technique was applied to improve detector performance when images contain partially occluded objects. In the last one, a score-level data fusion technique applied to encoded data. 
There are several natural extensions of this work:

1. In order to improve detection performance of a single-frame detector, a multiview detector could be developed by combining several view-specified classifiers. It would improve the detection performance of a single-frame detector significantly.

2. A set of Haar features could be extended to fit main features of targets.

3. The decision-level data fusion techniques such as Random Forest could be explored for improved automatic target detection.

4. The detector performance could be evaluated using a more realistic data and setup. We are currently in the process of building a database that will allow a more realistic setup and a more comprehensive analysis of the designed detector. 


\section{Bibliography}

[1] D. Hart and P. Craid-Hart, "Reducing swarming theory to practice for UAV control," Proc. IEEE Aerospace Conf., pp. 3050-3063, Mar. 2004.

[2] R. Blum, S. Kassam, and H. Poor, "Distributed detection with multiple sensors I: Advanced topics," Proc. IEEE, vol. 85, no. 1, pp. 64-79, Jan. 1997.

[3] X. Chen, S. Gong, N. Schmid, and M. Valenti, "UAV based distributed ATR under realistic simulated environmental effects," SPIE Defence and Security Symp., April 2007.

[4] P. Viola and M. Jones, "Rapid object detection using a boosted cascade of simple features," IEEE CVPR, pp. 1-9, 2001.

[5] R. Lienhart and J. Maydt, "An extended set of Haar-like features for rapid object detection," IEEE ICIP, vol. 1, no. 1, pp. 900-903, Sep. 2002.

[6] J. Dufour and V. Martin, "Active/passive cooperative image segmentation for automatic target recognition," SPIE, vol. 2294, pp. 552-560, 1994.

[7] M. Cohen, An Introduction to Automatic Target Recognition, EW Design Engineers Handbook, 1990.

[8] F. Gaudiano and E. Bonabeau, "Control of UAV swarms: What the bugs can teach us," Proc. of 2nd AIAA Unmanned Unlimited, pp. 582-589, 2003.

[9] H. Parunak J. Sauter, R. Matthews and S. Brueckner, "Performance of digital pheromones for swarming vehicle control," Proc. of AAMAS, pp. 903-910, 2005. 
[10] P. Dasgupta, "Distributed automatic target recognition using multi-agent UAV swarms," Proc. of AAMAS, pp. 479-481, 2006.

[11] M. Dorigo E. Bonabeau and G. Theraulaz, "Swarm intelligence: From natural to artificial systems," Oxford University Press, 1999.

[12] S. Edwards, "Swarming on the battlefield: Past, present and future," RAND National Security Research Div. Report, 2000.

[13] T. Leung, M. Burl, and P. Perona, "Finding faces in cluttered scenes using random labeled graph matching," Proc. Fifth IEEE ICCV, pp. 637-644, 1995.

[14] Y. Dai and Y. Nakano, "Face-texture model based on SGLD and its application in face detection in a color scene," Pattern Recognition, vol. 29, no. 6, pp. 10071017, 1996.

[15] M. Betke and N. Makris, "Fast object recognition in noisy images usng simulated annealing," Proceedings of the Fifth International Conference on Computer Vision, pp. 523-543, 1995.

[16] P. Hallinan A. Yuille and D. Cohen, "Feature extraction from faces using deformable templates," International Journal of Computer Vision, vol. 8, no. 2, pp. 99-111, 1992.

[17] S. Mallat, "A theory for multiresolution signal decomposition: The wavelet representation," IEEE Trans. Pattern Analysis and Machine Intelligence, vol. 11, no. 7, pp. 674-693, 1989.

[18] M. Turk and A. Pentland, "Eigenfaces for recognition," Technical Report-MIT, 1991.

[19] V. Vapnik, The Nature of Statistical Learning Theory, Springer Verlag, 1995.

[20] S. Baluj H. Rowley and T. Kanade, "Neural network-based face detection," IEEE Conference on Pattern Analysis and Machine Intelligence, vol. 20, no. 1, pp. 23-38, 1998. 
[21] Y. Freund and R. Schapire, "Experiments with a new boosting algorithm," Machine Learning: Proceedings of the Thirteenth International Conference, pp. 148-156, 1996.

[22] M. Oren, C. Papageorgiou, P. Sinha, E. Osuna, and T. Poggio, "Pedestrian detection using wavelet templates," Proc. Computer Vision and Pattern Recognition, pp. 193-199, 1997.

[23] C. Papageorgiou A. Mohan and T. Poggio, "Example-based object detection in images by components," IEEE Transactions on Pattern Analysis and Machine Intelligence, vol. 23, no. 4, pp. 349-361, 2001.

[24] C. Monrocq R. Vaillant and Y.L.Cun., "Original approach for the localisation of objects in images," IEEE Proc. Vis. Image Signal Processing, vol. 141, 1994.

[25] B. Moghaddam and A. Pentland, "Probabilistic visual learning for object detection," Technical Report MIT, vol. 326, 1995.

[26] H. Schneiderman and T. Kanade, "A statistical method for 3d object detection applied to faces and cars," IEEE Conference of Computer Vision and Pattern Recognition, pp. 1746-1751, 2000.

[27] R. Schapire and Y. Singer, "Improving boosting algorithms using confidencerated predictions," Machine Learning, vol. 37, no. 3, pp. 297-336, 1999.

[28] R. Schapire, Y. Freund, P. Bartlett, and W. Lee, "Boosting the margin: A new explanation for the effectiveness of voting methods," Annals of Statistics, vol. 26, pp. 1651-1686, 1998.

[29] F. Fleuret and D. Geman, "Coarse-to-fine face detection," Int. Journal of Computer Vision, vol. 41, no. 1, pp. 85-107, 2001.

[30] S. Agarwal A. Garg and T. Huang, "Fusion of global and local information for object detection," International Conference on Pattern Recognition, vol. 3, pp. 723-726, 2002. 
[31] S. Li, Z. Zhang, L. Zhu, and H. Zhang, "Real-time multi-view face detection," Fourth IEEE International Conference on Automatic Face and Gesture Recognition, vol. 4, pp. 67-81, 2002.

[32] M. Jones and P. Viola, "Fast multi-view face detection," Technical Report of Mitsubishi Electric Research Laboratories, 2003.

[33] E. Waltz and J. Llinas, Multisensor Data Fusion, Artech House, 1990.

[34] C. Chong, S. Mori, K. Chang, and W. Barker, "Architectures and algorithms for track association and fusion," IEEE Aerospace and Electronic Systems, vol. 15, no. 1, pp. 5-13, January.

[35] J. Aggarwal, Multisensor Fusion For Computer Vision, Springer-Verlag, 1993.

[36] H. Li, B. Manjunath, and S. Mitra, "Multi-sensor image fusion using the wavelet transform," ICIP, vol. 1, pp. 51-55, Nov. 1994.

[37] S. Nadimi and B. Bhanu, "Adaptive fusion for diurnal moving object detection," ICPR, vol. 3, pp. 696-699, Aug. 2004.

[38] M. Liggins and M. Nebrich, "Adaptive multi-image decision fusion," SPIE Signal Processing, Sensor Fusion, and Target Recognition IX, vol. 4052, pp. 218-228, Aug. 2000.

[39] Open Computer Vision Library:, ," http:/sourceforge.net/projects/opencvlibrary/.

[40] R. Lienhart, A. Kuranov, and V. Pisarevsky, "Empirical analysis of detection cascades of boosted classifiers for rapid object detection," MRL Technical Report, 2002.

[41] M. Park S. Park and M. Kang, "Super-resolution image reconstruction: A technical overview," IEEE Signal Processing Magazine, vol. 20, pp. 21-36, May 2003. 
[42] S. Borman and R. Stevenson, "Spatial resolution enhancement of low-resolution image sequences. A comprehensive review with directions for future research," Technical Report, University of Notre Dame, 1998.

[43] C. Kenney L. Fonseca, G.Hewer and B. Manjunath, "Registration and fusion of multispectral images using a new control point assessment method derived from optical flow ideas," Proc. SPIE Conference, vol. 3717, pp. 104-111, April 1999.

[44] M. Alam, J. Bognar, R. Hardie, and B. Yasuda, "Infrared image registration and high-resolution reconstruction using multiple translationally shifted aliased video frames," IEEE Trans. on Instrumentation and Measurement, vol. 49, pp. 915-923, Oct. 2000.

[45] C. Barber, D. Dobkin, and H. Huhdanpaa, "The quickhull algorithm of convex hulls," ACM Transactions on Mathematical Software, vol. 22, no. 4, pp. 469-483, Dec. 1996.

[46] H. L. Van Trees, Detection, Estimation, and Modulation Theory, Part I, John Wiley and Sons, New York, 2001.

[47] F. Sadjadi, "Application of genetic algorithm for automatic recognition of partially occluded objects," SPIE Automatic Object Recognition IV, vol. 2234, pp. 428-434, April 1994.

[48] S. Rees and B. Jones, "Operator for object recognition and scene analysis by estimation of a set occupancy with noisy and incomplete data sets," SPIE Intelligent Robots and Computer Vision XI, vol. 1825, pp. 289-297, November 1992.

[49] M. Hedley M. Trajkovic, "Fast corner detection," Image and Vision Computing, vol. 16, pp. 75-87, 1998.

[50] J. Kittler, M. Hatef, P. Duin, and J. Matas, "On combining classifiers," IEEE Trans. on Pattern Analysis and Machine Intelligence, vol. 20, pp. 226-239, March 1998. 
[51] L. Xu, A. Krzyzak, and C. Suen, "Methods of combining multiple classifiers and their applications to handwriting recognition," IEEE Trans. Systems, Man, and Cybernetics, vol. 22, no. 3, pp. 418-435, 1992.

[52] J. Kittleer, J. Matas, and K. Jonsson, "Combining evidence in personal identity verification systems," Pattern Recognition Letters, pp. 845-852, 1997.

[53] A. Savakis and H. Trussell, "Blur identification by residual spectral matching," IEEE Transactions on Image Processing, pp. 141-151, 1993.

[54] B. Bhanu, D. Dndgeon, E. Zelnio, A. Rosenfeld, D. Casasent, and I. Reed, "Introduction to the special issue on automatic target detection and recognition," IEEE Trans. Image Processing, vol. 6, no. 1, pp. 1-6, 1997.

[55] A. Lanterman, J. O'Sullivan, and M. Miller, "Kullback-Leibler distances for quantifying clutter and models," Optical Engineering, vol. 38, no. 12, pp. 21342146, 1999 . 